\title{
ON THE MAGNITUDES OF COMPACT SETS IN EUCLIDEAN SPACES
}

\author{
JUAN ANTONIO BARCELÓ AND ANTHONY CARBERY
}

\begin{abstract}
The notion of the magnitude of a metric space was introduced by Leinster in [11 and developed in [16, 12], 17, 20] and [13, but the magnitudes of familiar sets in Euclidean space are only understood in relatively few cases. In this paper we study the magnitudes of compact sets in Euclidean spaces. We first describe the asymptotics of the magnitude of such sets in both the small and large-scale regimes. We then consider the magnitudes of compact convex sets with nonempty interior in Euclidean spaces of odd dimension, and relate them to the boundary behaviour of solutions to certain naturally associated higher order elliptic boundary value problems in exterior domains. We carry out calculations leading to an algorithm for explicit evaluation of the magnitudes of balls, and this establishes the convex magnitude conjecture of Leinster and Willerton [12] in the special case of balls in dimension three. In general we show that the magnitude of an odd-dimensional ball is a rational function of its radius. In addition to Fourier-analytic and PDE techniques, the arguments also involve some combinatorial considerations.
\end{abstract}

\section{INTRODUCTION}

Motivated by considerations of a category-theoretic nature, Leinster [1] has introduced the notion of the magnitude of a metric space. Magnitude is an important new numerical invariant of a metric space which shares some of the more abstract properties of the Euler characteristic of a category (or of a topological space), and indeed both can be seen as special cases of the notion of the Euler characteristic or magnitude of an enriched category. In particular, the inclusion-exclusion principle enjoyed by the Euler characteristic provides important motivation for the hopedfor properties of magnitude. More generally, magnitude is designed to capture the "essential size" of a metric space in a more subtle way than cruder measures such as cardinality or diameter, and at the same time it will also contain further significant geometric information concerning the space. For a much more detailed discussion of these issues see [11, [12, 20] and [13].

Leinster's definition of the magnitude of a finite metric space bears close resemblance to notions of a potential-theoretic nature, and Meckes [16] and [17] has developed this perspective to the point where a tractable definition of the magnitude of a positive-definite compact metric space can now be given in terms analogous to those of classical capacity. This provides the starting point for our investigations.

Before describing our results, we give a little more informal background on magnitude in order that our contributions can be placed in context.

Date: 9th July 2015, revised 13th July 2016.

The first author was supported by Spanish Grant MTM2011-28198. 
1.1. Definitions of magnitude and connection with capacity. Given a finite metric space $(X, d)$, Leinster [11] defined its magnitude as the value

$$
|X|=\sum_{x \in X} w(x)
$$

whenever $w: X \rightarrow \mathbb{R}$ satisfies

$$
\sum_{y \in X} e^{-d(x, y)} w(y)=1
$$

for all $x \in X$.

It is easy to check that any two such $w$ will give the same value for the magnitude, and if no such $w$ exists we declare the magnitude to be undefined. Under the mild additional condition that $X$ be positive-definite (meaning that the matrix $\left(e^{-d(x, y)}\right)_{x, y \in X}$ is positive-definite), its magnitude is defined, see [11, [16] and [17. Finite subsets of Euclidean spaces are always positive-definite, [11. It is easy to check (see below for the argument) that $|\emptyset|=0,|\{x\}|=1$ and that if $X_{N}$ is an $N$-point space consisting of the vertices of a simplex in $\mathbb{R}^{n}$ (with $n \geq N-1$ ) which are all equidistant $t$ from one another, then

$$
\left|X_{N}\right|=\frac{N}{1+(N-1) e^{-t}} .
$$

We describe a compact metric space as positive-definite if every finite subset is positive-definite, and the magnitude of a compact positive-definite metric space $(X, d)$ has been defined [16] as

$$
|X|=\sup \{|\Xi|: \Xi \text { a finite subset of } X\} .
$$

(Once again, any compact subset of a Euclidean space is positive-definite.)

Bearing in mind Leinster's definition, it is natural to consider the class of finite signed Borel measures $\mu$ on a compact positive-definite metric space $X$, and for such a $\mu$ introduce its potential function $\Phi_{\mu}$ given by

$$
\Phi_{\mu}(x):=\int_{X} e^{-d(x, y)} \mathrm{d} \mu(y) .
$$

If there is such a finite signed Borel measure $\mu$ on $X$ satisfying

$$
\Phi_{\mu}(x)=\int_{X} e^{-d(x, y)} \mathrm{d} \mu(y) \equiv 1 \text { on } X,
$$

then $\mu$ is called a weight measure for $X$. It is known ([16, [17]) that if $X$ is compact and positive-definite, then $|X|=\mu(X)$ for any weight measure $\mu$.

If we take $X=[-R, R] \subseteq \mathbb{R}$ with the usual metric, then one simply checks using integration by parts that, with $\mathrm{d} x$ denoting Lebesgue measure on $\mathbb{R}, \frac{1}{2}\left(\delta_{-R}+\delta_{R}+\right.$ $\left.\mathrm{d} x\right|_{[-R, R]}$ ) is a weight measure for $X$, and hence $|[-R, R]|=R+1$. (See [11] and [17.) This is the only example of a nontrivial compact convex set in a Euclidean space whose magnitude was hitherto known.

In nearly all examples for which the magnitude is known explicitly one can fairly easily identify a weight measure; in particular when the metric space $X$ enjoys a lot of symmetry one expects a weight measure to reflect this symmetry and this leads to a limited range of possibilities. For example, in the case of the simplex mentioned above, we just set $w(y)=a$ for all $y$ and observe that the equation 
$\sum_{y \in X} e^{-d(x, y)} w(y)=1$ becomes $a+(N-1) e^{-t} a=1$, leading to (11). For more examples see [11, 12, 20. Our approach here is different and is motivated by connections with differential equations.

There is a clear analogy between magnitude and the notion of capacity as developed in classical potential theory, and which we now describe in a very informal manner. A possible definition of the $\alpha$-capacity of a compact metric space is

$$
\operatorname{cap}_{\alpha}(X)=\sup \mu(X)
$$

where the sup is taken over all positive finite Borel measures $\mu$ on $X$ such that

$$
\int_{X} d(x, y)^{-\alpha} \mathrm{d} \mu(y) \leq 1 \text { on } X .
$$

The study of the potentials $\int_{X} d(x, y)^{-\alpha} \mathrm{d} \mu(y)$ and the associated $\alpha$-capacities has a long and distinguished history. When we are in Euclidean space $\mathbb{R}^{n}$ with the usual metric, we can take advantage of the Fourier transform to characterise the $\alpha$-capacity of a compact subset $X$ for $0<\alpha<n$ as

$$
\operatorname{cap}_{\alpha}(X)=c_{n, \alpha} \inf \left\{\int_{\mathbb{R}^{n}}|\xi|^{n-\alpha}|\widehat{f}(\xi)|^{2} \mathrm{~d} \xi: f \geq 1 \text { on } X\right\}
$$

where $c_{n, \alpha}$ is a certain dimensional constant and ${ }^{\wedge}$ denotes the Fourier transform. See for example [1] for a thorough and detailed discussion of potential theory and capacity in the Euclidean setting.

Meckes in [17] develops the analogy between magnitude and capacity beyond the formal level, and gives an extremal characterisation of magnitude in some generality. In the case of compact sets $X$ in Euclidean space $\mathbb{R}^{n}$ this characterisation can be realised as

$$
|X|=\frac{1}{n ! \omega_{n}} \inf \left\{\|f\|_{H^{(n+1) / 2}\left(\mathbb{R}^{n}\right)}^{2}: f \in H^{(n+1) / 2}\left(\mathbb{R}^{n}\right), f \equiv 1 \text { on } X\right\}
$$

where $\omega_{n}$ is the volume of the unit ball in $\mathbb{R}^{n}$ and $H^{m}\left(\mathbb{R}^{n}\right)$ is the Sobolev space of functions whose derivatives of order up to $m$ are in $L^{2}\left(\mathbb{R}^{n}\right)$. More precisely, $H^{m}\left(\mathbb{R}^{n}\right)$ is the space of Bessel potentials of order $m$, and its norm is given by

$$
\|f\|_{H^{m}\left(\mathbb{R}^{n}\right)}^{2}:=\left\|(I-\Delta)^{m / 2} f\right\|_{L^{2}\left(\mathbb{R}^{n}\right)}^{2} .
$$

(There are many different - but equivalent - norms on the space $H^{m}\left(\mathbb{R}^{n}\right)$ which are regularly employed in the literature, especially when $m$ is an integer. We emphasise that throughout this paper, we exclusively use the definition above.) Note that when $m=(n+1) / 2>n / 2$, functions in $H^{m}$ are continuous by the Sobolev embedding theorem so the prescription $f \equiv 1$ on $X$ makes sense pointwise.

One can informally motivate this result of Meckes in line with classical potential theory as follows. Adopting the standard convention from Fourier analysis - which differs from that used by Meckes in [16] and [17] - that the Fourier transform is given by

$$
\widehat{f}(\xi)=\int_{\mathbb{R}^{n}} f(x) e^{-2 \pi i x \cdot \xi} \mathrm{d} x,
$$

then it defines an isometry on $L^{2}$, and we have

$$
\widehat{e^{-|\cdot|}}(\xi)=n ! \omega_{n}\left(1+4 \pi^{2}|\xi|^{2}\right)^{-\frac{n+1}{2}} \text {. }
$$


(See for example Stein, [18.) So if $\mu$ is a weight measure for $X \subseteq \mathbb{R}^{n}$ we can extend it to be a measure on the whole of $\mathbb{R}^{n}$ in the canonical way, $\Phi_{\mu}$ becomes defined on all of $\mathbb{R}^{n}$ and we have $\Phi_{\mu}(x)=1$ on $X$. On the other hand, by taking Fourier transforms,

$$
\widehat{\Phi_{\mu}}(\xi)=n ! \omega_{n}\left(1+4 \pi^{2}|\xi|^{2}\right)^{-\frac{n+1}{2}} \widehat{\mu}(\xi)
$$

Therefore

$$
\begin{aligned}
\mu(X) & =\int_{X} \Phi_{\mu}(x) \mathrm{d} \mu(x)=\int_{\mathbb{R}^{n}} \int_{\mathbb{R}^{n}} e^{-|x-y|} \mathrm{d} \mu(y) \mathrm{d} \mu(x) \\
& =\int_{\mathbb{R}^{n}} n ! \omega_{n}\left(1+4 \pi^{2}|\xi|^{2}\right)^{-\frac{n+1}{2}}|\widehat{\mu}(\xi)|^{2} \mathrm{~d} \xi \\
& =\frac{1}{n ! \omega_{n}} \int_{\mathbb{R}^{n}}\left|\widehat{\Phi_{\mu}}(\xi)\right|^{2}\left(1+4 \pi^{2}|\xi|^{2}\right)^{\frac{n+1}{2}} \mathrm{~d} \xi
\end{aligned}
$$

and this last expression is exactly $\frac{1}{n ! \omega_{n}}\left\|\Phi_{\mu}\right\|_{H^{(n+1) / 2}\left(\mathbb{R}^{n}\right)}^{2}$.

It is easy to see using Plancherel's theorem and the binomial theorem that when $m$ is an integer the inner product $\langle\cdot, \cdot\rangle_{H^{m}\left(\mathbb{R}^{n}\right)}$ corresponding to the $H^{m}$ norm is given by

$$
\langle f, g\rangle_{H^{m}\left(\mathbb{R}^{n}\right)}:=\sum_{j=0}^{m}\left(\begin{array}{c}
m \\
j
\end{array}\right) \int_{\mathbb{R}^{n}} D^{j} f \cdot \overline{D^{j} g}
$$

where $D^{j} f=\Delta^{j / 2} f$ for $j$ even and $D^{j} f=\nabla \Delta^{(j-1) / 2} f$ for $j$ odd. We shall make systematic use of this in what follows.

One should note that although the right-hand side of equation (2) apparently depends upon the Euclidean space in which $X$ sits, the original definition of the magnitude of $X$ is an intrinsic metric invariant, and so formula (2) will hold for whatever Euclidean space $\mathbb{R}^{n}$ in which we can embed $X$.

1.2. Magnitude and geometric invariants. It is perhaps not a priori clear why, despite its ubiquity, the exponential function $s \mapsto e^{-s}$ is singled out to appear in the definition of magnitude in composition with the metric. Ultimately the reason for this is that its key property of converting addition to multiplication distinguishes it as the (essentially) unique function $\Psi$ such that, if $\Psi(d(x, y))$ is the kernel of the potential function, then the magnitude of a finite metric space (as defined via $\Psi$ ) and the Euler characteristic of a finite category share a common generalisation in the Euler characteristic or magnitude of an enriched category. In particular, with $\Psi(s)=e^{-s}$, the triangle inequality gives us

$$
\Psi(d(x, y)) \geq \Psi(d(x, z)) \Psi(d(z, y))
$$

for all $x, y, z \in X$; this inequality is to be thought of as analogous to the categorytheoretic composition of a map from $x$ to $z$ with a map from $z$ to $y$ to obtain a map from $x$ to $y$. For more details see [11.

In the category of finite sets, magnitude is just cardinality, which obviously satisfies the familiar inclusion-exclusion principle

$$
\#(A \cup B)+\#(A \cap B)=\#(A)+\#(B) .
$$


Similarly, in the category of finite simplicial complexes, the classical Euler characteristic $\chi$ can be characterised by $\chi(\emptyset)=0, \chi(\{x\})=1$ and

$$
\chi(A \cup B)+\chi(A \cap B)=\chi(A)+\chi(B) .
$$

However, magnitude in the setting of finite metric spaces is manifestly not finitely additive as the example of the simplex with equidistant vertices, (1), indicates. Nevertheless, formula (11) tells us that as $t \rightarrow \infty$, the magnitude of $X_{N}$ does asymptotically satisfy the inclusion-exclusion principle since $\left|X_{N}\right|$ approaches $N$ as $t \rightarrow \infty$. More generally if $(X, d)$ is any finite metric space and if we let $t X=(X, t d)$ for $t>0$, it is known (see [11) that $|t X| \rightarrow \# X$ as $t \rightarrow \infty$, and so inclusion-exclusion holds in an asymptotic sense for the class of finite metric spaces. On the other hand, if we consider the (admittedly somewhat limited) class $\mathcal{K}$ of compact convex sets in $\mathbb{R}$, then if $A, B \in \mathcal{K}$ are such that $A \cup B \in \mathcal{K}$, we have the exact inclusion-exclusion identity

$$
|A \cup B|+|A \cap B|=|A|+|B|
$$

for magnitude (since the compact interval $[a, b]$ has magnitude $1+(b-a) / 2$ as we have seen above). These considerations lead one to hope that magnitude in general will satisfy the inclusion-exclusion principle in some asymptotic sense, and perhaps exactly so under certain more restricted circumstances - such as in the presence of convexity. (One may remark that the classical theory of capacity of compact convex sets is richer than the general theory, and from the category-theoretic point of view convex sets are natural in so far as the path of minimal distance between any two points is contained in the set.)

Examples of metric spaces in which there is a lot of symmetry, and therefore where it is possible to evaluate the magnitude directly, yield further insight into some of the geometric characteristics which magnitude might capture. In analogy with the calculation for the simplex above, if $X$ is a compact metric space and $G$ is a compact group with Haar measure $\nu$, acting transitively and isometrically on $X$, then the only sensible candidate for a weight measure for $t X=(X, t d)$ should have constant density $\lambda a(t)$ (for some $\lambda>0$ ) where $a(t) \int_{G} e^{-t d(x, g y)} \mathrm{d} \nu(g)=1$ (the integral is independent of $x$ and $y$ ). So the magnitude of $t X$ should be

$$
|t X|=\frac{\nu(G)}{\int_{G} e^{-t d(x, g y)} \mathrm{d} \nu(g)} .
$$

Exact evaluation or asymptotic analysis of such expressions as $t \rightarrow \infty$ has led to the following results:

Theorem A (Willerton, 20].) Let $X$ be a two-dimensional homogeneous compact manifold. Then

$$
|t X|=\frac{1}{2 \pi} \operatorname{Area}(X) t^{2}+\chi(X)+O\left(t^{-2}\right)
$$

as $t \rightarrow \infty$, where $\chi$ is the classical Euler characteristic.

Theorem B (Willerton, [20].) Let $n \geq 1$. Then there is an explicit formula for $\left|t \mathbb{S}^{n}\right|$ with its geodesic metric, which yields

$$
\left|t \mathbb{S}^{n}\right|=\frac{1}{n ! \omega_{n}} \operatorname{Vol}_{n}\left(\mathbb{S}^{n}\right) t^{n}+\frac{n+1}{6 n ! \omega_{n}} \operatorname{tsc}\left(\mathbb{S}^{n}\right) t^{n-2}+\cdots+\chi\left(\mathbb{S}^{n}\right)+O\left(e^{-t}\right)
$$

as $t \rightarrow \infty$, where $\mathrm{Vol}_{n}$ denotes $n$-dimensional volume, $\chi$ is the classical Euler characteristic and tsc is the total scalar curvature. 
(Note that $\chi\left(\mathbb{S}^{n}\right)$ is 2 for $n$ even and 0 for $n$ odd.) Willerton also has formulae for the magnitude of spheres in Euclidean spaces with the subspace metric which are explicit when $n=1$ and 2 and give the first few terms in the asymptotic expansion as $t \rightarrow \infty$ for larger values of $n$. In addition, he has obtained the first four terms in the asymptotic expansion for a general $n$-dimensional homogeneous compact manifold.

Together with the result that for a nonempty compact interval $I \subseteq \mathbb{R}$ we have

$$
|t I|=\frac{1}{2} \operatorname{Length}(I) t+\chi(I)
$$

(where $\chi(I)$, the Euler characteristic of $I$, equals 1 ), these results of Willerton suggest that $t \mapsto|t X|$ should have a polynomial flavour as $t \rightarrow \infty$, with the coefficients of the polynomial part encapsulating important geometric information concerning $X$. Combined with the results of numerical calculations 19 and some parallel results when the $l^{1}$-metric is used instead of the usual metric (see [11]), they provide evidence for the following conjectures of Leinster and Willerton from [12]:

Conjecture 1 (Leinster-Willerton). For suitable (i.e. so that perimeter and classical Euler characteristic are at least defined) compact $X \subseteq \mathbb{R}^{2}$ we have, as $t \rightarrow \infty$,

$$
|t X|=\frac{1}{2 \pi} \operatorname{Area}(X) t^{2}+\frac{1}{4} \operatorname{Perim}(X) t+\chi(X)+o(1) .
$$

Here we see that intermediate-dimensional geometrical characteristics (in this case the perimeter) of $X$ are expected to play a role. We shall not be directly concerned with Conjecture 1 in the current paper, but will instead focus on the following, known as the convex magnitude conjecture of Leinster and Willerton, [12]:

Conjecture 2 (Leinster-Willerton). Suppose $X \subseteq \mathbb{R}^{n}$ is compact and convex. Then $t \mapsto|t X|$ is a polynomial of degree $n$ and moreover

$$
|t X|=\frac{\operatorname{Vol}(X)}{n ! \omega_{n}} t^{n}+\frac{\operatorname{Surf}(\partial X)}{2(n-1) ! \omega_{n-1}} t^{n-1}+\cdots+1=\sum_{i=0}^{n} \frac{1}{i ! \omega_{i}} V_{i}(X) t^{i}
$$

where $V_{i}(X)$ is the $i$ 'th intrinsic volume of $X$ and $\omega_{i}$ is the volume of the unit ball in $\mathbb{R}^{i}$.

The intrinsic volumes $V_{i}$ are classical integral-geometric invariants defined on the class of compact convex sets $\mathcal{K}$ in Euclidean space, and $V_{i}(X)$ encapsulates the quantitative $i$-dimensional information concerning $X$. In particular we have $V_{i}(t X)=$ $t^{i} V_{i}(X)$. Indeed, $V_{n}$ is ordinary volume, $V_{n-1}$ is half the surface area, and $V_{i}$ for $1 \leq i \leq n-2$ captures the $i$-dimensional information on the boundary $\partial X$, while $V_{0}(X)=1$ for nonempty $X$ and $V_{0}(X)=0$ when $X$ is empty. Each $V_{i}$ is a valuation on $\mathcal{K}$, which means that $V_{i}(\emptyset)=0$, and if $A, B \in \mathcal{K}$ are such that $A \cup B \in \mathcal{K}$, then we have the inclusion-exclusion identity

$$
V_{i}(A \cup B)+V_{i}(A \cap B)=V_{i}(A)+V_{i}(B) .
$$

For more detail see [10]. So the convex magnitude conjecture implies in particular that magnitude is a valuation on $\mathcal{K}$. On the other hand, it is known by Hadwiger's theorem (see again [10]) that any valuation on $\mathcal{K}$ which is continuous with respect to the Hausdorff metric and which is invariant under Euclidean motions must be given by a linear combination of the intrinsic volumes. It is also known (see [14, 15]) that 
a valuation which is translation-invariant and monotone is necessarily continuous. Clearly magnitude is invariant under Euclidean motions and is monotone, so the convex magnitude conjecture is true if and only if magnitude is a valuation and the conjecture is true for a suitable family of compact convex sets - such as the closed balls - which serves to normalise the precise coefficients of the polynomial arising. It is therefore of considerable interest to determine the validity of the conjecture in the special case of closed balls in $\mathbb{R}^{n}$, and this issue is the main focus of the present paper.

On the other hand, one does not see the inclusion-exclusion principle arising in classical potential theory, so perhaps a certain amount of caution is in order in approaching this issue.

To fix ideas, the convex magnitude conjecture predicts that the magnitude of the closed ball $B_{R}$ of radius $R$ in $\mathbb{R}^{n}$ will be:

$$
\begin{aligned}
& n=1: \quad R+1 \\
& n=2: \quad \frac{R^{2}}{2 !}+\frac{\pi R}{2}+1 \\
& n=3: \quad \frac{R^{3}}{3 !}+R^{2}+2 R+1 \\
& n=4: \quad \frac{R^{4}}{4 !}+\frac{\pi R^{3}}{8}+\frac{3 R^{2}}{2}+\frac{3 \pi R}{4}+1 \\
& n=5: \quad \frac{R^{5}}{5 !}+\frac{R^{4}}{9}+\frac{2 R^{3}}{3}+2 R^{2}+\frac{8 R}{3}+1 \\
& n=6: \quad \frac{R^{6}}{6 !}+\frac{\pi R^{5}}{128}+\frac{5 R^{4}}{24}+\frac{5 \pi R^{3}}{16}+\frac{5 R^{2}}{2}+\frac{15 \pi R}{16}+1 \\
& n=7: \quad \frac{R^{7}}{7 !}+\frac{R^{6}}{225}+\frac{R^{5}}{20}+\frac{R^{4}}{3}+\frac{4 R^{3}}{3}+3 R^{2}+\frac{16 R}{5}+1,
\end{aligned}
$$

etc.

1.2.1. What is currently known about the convex magnitude conjecture. It is clear from (2) that $t \mapsto|t X|$ is an increasing function of $t$ when $X$ is compact and convex. Meckes [17] has shown that this function is continuous on $(0, \infty)$, and Leinster [11] (see also Meckes [17]) has shown that for arbitrary compact sets in $\mathbb{R}^{n}$ we have

$$
|X| \geq \frac{\operatorname{Vol}(X)}{n ! \omega_{n}}
$$

When $n=1$ the conjecture is true (see above), but when $n \geq 2$, methods based entirely on symmetry - and indeed on weight measures - will not suffice to resolve it, and instead we turn to techniques of differential equations.

We remark that a natural analogue of the convex magnitude conjecture does hold for convex bodies in $\mathbb{R}^{n}$ when instead of the $e l l^{2}$ norm we use the $\ell_{1}^{n}$ norm. See Theorem $4.6(2)$ of $[13$.

1.3. Main results of the present paper. We shall first establish that the asymptotics as $t \rightarrow 0$ and $t \rightarrow \infty$ which are predicted by the Leinster-Willerton convex magnitude conjecture do indeed hold, and in fact do so more generally for nonempty compact sets. That is, we prove: 
Theorem 1. Let $X$ be a nonempty compact set in $\mathbb{R}^{n}$. Then

$$
|R X| \rightarrow 1 \text { as } R \rightarrow 0
$$

and

$$
R^{-n}|R X| \rightarrow \frac{\operatorname{Vol}(X)}{n ! \omega_{n}} \text { as } R \rightarrow \infty .
$$

As a consequence of the first statement we have that $t \mapsto|t X|$ is continuous also at $t=0$.

We shall next be concerned with the validity of the convex magnitude conjecture for the class of convex bodies in Euclidean space $\mathbb{R}^{n}$, that is, compact convex sets with nonempty interior. Our starting point will be Meckes' formula (2) for the magnitude of such a convex body and we shall try to relate this to the formula (5) appearing in the convex magnitude conjecture.

Meckes has already observed [17 that the extremiser in (2) exists and is unique, and also satisfies the associated Euler-Lagrange equation

$$
(I-\Delta)^{(n+1) / 2} f=0 \text { weakly on } \mathbb{R}^{n} \backslash X
$$

which, when $n$ is odd, is an elliptic differential (as opposed to pseudodifferential) equation. In order to be able to work with differential rather than pseudodifferential equations we shall from Section 7 onwards assume that $n$ is odd. We shall first show that there is a unique member of $H^{(n+1) / 2}\left(\mathbb{R}^{n}\right)$ which is a solution to (6) and which is identically 1 on $X$. This solution therefore extremises the energy $\|f\|_{H^{(n+1) / 2}\left(\mathbb{R}^{n}\right)}^{2}$ over all $f \in H^{(n+1) / 2}\left(\mathbb{R}^{n}\right)$ with $f \equiv 1$ on $X$.

The convex magnitude conjecture predicts a single term corresponding to the volume of $X$, with the remaining terms mainly relating to the boundary $\partial X$. Our next task is therefore to develop a formula for the extremal energy expressed in terms of $\operatorname{Vol}(X)$ and the values of the extremising function near $\partial K$. This is carried out in order to highlight the contribution to the extremal energy arising from the boundary of $X$ and hence ultimately to facilitate a comparison between (2) and (5). The formula is analogous to classical representations of capacities as boundary integrals of functions of solutions to the associated partial differential equations, and it appears in Theorem 5 below.

We then turn to the problem of explicit identification of the extremiser in the case that $X$ is a nontrivial closed ball. This is achieved by using spherical symmetry to reduce matters to consideration of certain ordinary differential equations.

Finally, having obtained the extremiser, we use our formula from Theorem 5 to obtain an explicit evaluation of the magnitude of a closed ball. (Strictly speaking we could in principle avoid the use of our formula at this stage and instead simply calculate $\|f\|_{H^{(n+1) / 2\left(\mathbb{R}^{n}\right)}}^{2}$ directly for the extremiser, but we wish to try to identify precisely the contributions of the boundary of the ball to its magnitude, and in any case application of the formula is fairly direct.) Leinster and Meckes [13] have recently developed an alternate approach to calculation of $\|f\|_{H^{(n+1) / 2}\left(\mathbb{R}^{n}\right)}^{2}$ which bypasses our Theorem 5 , but which does not emphasise the role of the boundary. See the remark at the end of Section 5 .

In fact, the explicit identification of the extremiser is not an entirely straightforward process. Our procedures amount to giving an algorithm for its identification in any 
odd dimension, and thus yield an algorithm for the formula for the magnitude of a closed ball in any odd dimension. The formulae become more complex as dimension increases and so we provide explicit versions only in dimensions 1, 3, 5 and 7.

The first upshot of these calculations is that the Leinster-Willerton convex magnitude conjecture is true for closed balls in three dimensions:

Theorem 2. The magnitude of the closed ball of radius $R>0$ in $\mathbb{R}^{3}$ is

$$
\frac{R^{3}}{3 !}+R^{2}+2 R+1
$$

However, in higher odd dimensions the magnitude is not a polynomial in the radius, thus disproving the convex magnitude conjecture in general. In dimension five we have:

Theorem 3. The magnitude of the closed ball of radius $R>0$ in $\mathbb{R}^{5}$ is

$$
\frac{R^{5}}{5 !}+\frac{3 R^{5}+27 R^{4}+105 R^{3}+216 R^{2}+72}{24(R+3)} .
$$

Note that when $R=0$ this expression takes the value 1 , and as $R \rightarrow \infty$ the leading term is $R^{5} / 5$ !, but the formula does not agree with the conjectured value of

$$
\frac{R^{5}}{5 !}+\frac{R^{4}}{9}+\frac{2 R^{3}}{3}+2 R^{2}+\frac{8 R}{3}+1
$$

If one expands the expression for the magnitude asymptotically for $R>3$ one obtains

$$
\frac{R^{5}}{5 !}+\frac{R^{4}}{8}+\frac{3 R^{3}}{4}+\frac{17 R^{2}}{8}+\frac{21 R}{8}+\frac{9}{8}+O\left(\frac{1}{R}\right)
$$

as $R \rightarrow \infty$. (Notice that some, but not all, of the coefficients here differ by a multiplicative factor of $9 / 8$ 'ths from the conjectured values.) The role of the value $R=-3$ remains mysterious.

Although the magnitude is not a polynomial for odd $n \geq 5$, it is the next best thing:

Theorem 4. The magnitude of the closed ball of radius $R>0$, in a Euclidean space $\mathbb{R}^{n}$ of odd dimension, is a rational function of $R$ with rational coefficients.

It seems possible that one might be able to take the coefficients in the polynomials featuring in the rational function to be nonnegative, but this has not been verified in general. One can show that the denominator can be taken to be a polynomial of degree strictly less than $\frac{3 n^{2}-2 n+7}{8}$.

There remain the tantalising questions of whether magnitude is a valuation in dimension three - and indeed whether the convex magnitude conjecture is true in general in dimension three - and of what happens in even dimensions, especially dimensions two and four. Is $|R X|$ a rational function of $R$ for general compact convex bodies $X$ in odd dimensions? If so, do the coefficients of the polynomials have a geometric significance? (See Section 10 for a brief discussion of cuboids and ellipsoids in three-dimensional space.) 
1.4. Structure of the paper. The paper falls into three main parts.

In the first part, in Section 2 we begin by giving the asymptotic results of Theorem 1. based on little more than elementary Fourier analysis.

In the second part we develop the general PDE theory for our problem. In Section 3 we set out the basic facts about Sobolev spaces which we shall need. In Section [4 we formulate the variational problem for whose solution we develop a formula in Section 5

In the third part we focus on the spherically symmetric situation. In Section 6 we give some combinatorial preliminaries. In Section 7 we find the general solution of the PDE problem from Section 4 in the spherically symmetric case. In Section 8 we fit the boundary conditions and develop an algorithm to identify our explicit solution and its magnitude. The discussion here leads to the proof of Theorem 4 in Subsection 8.4. In Section 9 we implement the algorithm in dimensions 1, 3, 5 and 7 , leading to Theorems 2 and 3 .

Finally, in Section 10 we make some concluding remarks.

1.5. Acknowledgements. The authors would like to thank Tom Leinster for introducing them to the fascinating and beautiful subject of magnitude, and for patiently and carefully explaining its category-theoretic origins in a series of seminars and other discussions at the University of Edinburgh. They would also like to thank Mark Meckes for a number of illuminating email exchanges and comments. Finally, they would like to thank the referees whose careful reading of the manuscript has led to several clarifications and presentational improvements.

\section{AsYmptotic RESUlts}

We prove the asymptotic statements of Theorem 1 which are based on elementary Fourier analysis.

Let us first consider the asymptotic behaviour as $R \rightarrow \infty$. If $f \in H^{(n+1) / 2}\left(\mathbb{R}^{n}\right)$ satisfies $f \equiv 1$ on a compact set $X$, it must manifestly satisfy $\|f\|_{H^{(n+1) / 2}\left(\mathbb{R}^{n}\right)}^{2} \geq$ $\|f\|_{L^{2}\left(\mathbb{R}^{n}\right)}^{2} \geq \operatorname{Vol}(X)$, so that

$$
|X| \geq \frac{\operatorname{Vol}(X)}{n ! \omega_{n}}
$$

as has been observed by Leinster [11] and Meckes [17. But also

$$
\begin{aligned}
\left\|f\left(R^{-1} \cdot\right)\right\|_{H^{(n+1) / 2}\left(\mathbb{R}^{n}\right)}^{2} & =\int_{\mathbb{R}^{n}}\left|R^{n} \widehat{f}(R \xi)\right|^{2}\left(1+4 \pi^{2}|\xi|^{2}\right)^{(n+1) / 2} \mathrm{~d} \xi \\
& =R^{n} \int_{\mathbb{R}^{n}}|\widehat{f}(\xi)|^{2}\left(1+4 \pi^{2}(|\xi| / R)^{2}\right)^{(n+1) / 2} \mathrm{~d} \xi
\end{aligned}
$$

so that, by the monotone (or dominated) convergence theorem,

$$
R^{-n}\left\|f\left(R^{-1} \cdot\right)\right\|_{H^{(n+1) / 2}\left(\mathbb{R}^{n}\right)}^{2} \rightarrow \int_{\mathbb{R}^{n}}|\widehat{f}(\xi)|^{2} \mathrm{~d} \xi=\int_{\mathbb{R}^{n}}|f(x)|^{2} \mathrm{~d} x
$$

as $R \rightarrow \infty$. So we have

$$
\frac{\operatorname{Vol}(X)}{n ! \omega_{n}} \leq R^{-n}|R X| \leq \frac{R^{-n}\left\|f\left(R^{-1} \cdot\right)\right\|_{H^{(n+1) / 2}\left(\mathbb{R}^{n}\right)}^{2}}{n ! \omega_{n}} \rightarrow \frac{\int_{\mathbb{R}^{n}}|f(x)|^{2} \mathrm{~d} x}{n ! \omega_{n}}
$$


using (7), (2) and (8) successively. Now we can find $f \in H^{(n+1) / 2}\left(\mathbb{R}^{n}\right)$ with $f \equiv 1$ on $X$ such that $\|f\|_{2}^{2}$ is as close as we like to $\operatorname{Vol}(X)$. Indeed, with $\Phi: \mathbb{R}^{n} \rightarrow$ $[0, \infty)$ a smooth function of compact support in $\{|x| \leq 1\}$ with $\int \Phi=1, \Phi_{r}(x):=$ $r^{-n} \Phi\left(r^{-1} x\right)$ and $X_{r}:=\left\{x \in \mathbb{R}^{n}: d(x, X) \leq r\right\}$ we have that $f_{r}:=\Phi_{r} * \chi_{X_{r}}$ is a nonnegative smooth function of compact support which satisfies $f_{r}(x)=1$ for $x \in X$, and $f_{r}(x) \rightarrow 0$ as $r \rightarrow 0$ for $x \notin X$. So by the dominated convergence theorem, $\int f_{r}^{2} \rightarrow \operatorname{Vol}(X)$ as $r \rightarrow 0$. Therefore

$$
R^{-n}|R X| \rightarrow \frac{\operatorname{Vol}(X)}{n ! \omega_{n}}
$$

as $R \rightarrow \infty$.

Now let us consider what happens as $R \rightarrow 0$. Note that we may assume that $n$ is odd since as we remarked above in Section 1, magnitude is intrinsically defined.

If $f \in H^{(n+1) / 2}\left(\mathbb{R}^{n}\right)$ and $f(0)=1$, then

$$
\begin{gathered}
1=|f(0)| \leq \sup _{x}|f(x)| \leq\|\widehat{f}\|_{L^{1}\left(\mathbb{R}^{n}\right)} \\
\leq\left(\int|\widehat{f}(\xi)|^{2}\left(1+4 \pi^{2}|\xi|^{2}\right)^{(n+1) / 2} \mathrm{~d} \xi\right)^{1 / 2}\left(\int \frac{\mathrm{d} \xi}{\left(1+4 \pi^{2}|\xi|^{2}\right)^{(n+1) / 2}}\right)^{1 / 2}
\end{gathered}
$$

so that

$$
\|f\|_{H^{(n+1) / 2}\left(\mathbb{R}^{n}\right)}^{2} \geq\left(\int \frac{\mathrm{d} \xi}{\left(1+4 \pi^{2}|\xi|^{2}\right)^{(n+1) / 2}}\right)^{-1}
$$

with equality if and only if $\widehat{f}(\xi)$ is the scalar multiple of $\left(1+4 \pi^{2}|\xi|^{2}\right)^{-(n+1) / 2}$ with $f(0)=1$, or, equivalently, $f(x)=e^{-|x|}$. By (3) and Fourier inversion,

$$
\int \frac{\mathrm{d} \xi}{\left(1+4 \pi^{2}|\xi|^{2}\right)^{(n+1) / 2}}=\frac{1}{n ! \omega_{n}} \int \widehat{e^{-|\cdot|}}(\xi) \mathrm{d} \xi=\frac{1}{n ! \omega_{n}} e^{-|0|}=\frac{1}{n ! \omega_{n}}
$$

so that

$$
\|f\|_{H^{(n+1) / 2\left(\mathbb{R}^{n}\right)}}^{2} \geq n ! \omega_{n}
$$

(with equality if and only if $f(x)=f_{0}(x):=e^{-|x|}$ ). This implies that $|X| \geq 1$ so long as $X \neq \emptyset$.

Now, for $0<R \leq 1$, let $f_{R}: \mathbb{R}^{n} \rightarrow[0,1]$ be a smooth function which satisfies

$$
f_{R}(x)= \begin{cases}1, & |x| \leq R \\ e^{R} e^{-|x|}, & |x| \geq R^{1 / 2}\end{cases}
$$

and, for $R \leq|x| \leq R^{1 / 2}$ and $|\alpha| \geq 1$,

$$
\left|\left(\frac{\partial}{\partial x}\right)^{\alpha} f_{R}(x)\right| \lesssim R^{-(|\alpha|-1) / 2} .
$$

We then have, (see (4)),

$$
\begin{gathered}
\left\|f_{R}\right\|_{H^{(n+1) / 2}\left(\mathbb{R}^{n}\right)}^{2}=\sum_{j=0}^{(n+1) / 2}\left(\begin{array}{c}
\frac{n+1}{2} \\
j
\end{array}\right) \int_{\mathbb{R}^{n}}\left|D^{j} f_{R}(x)\right|^{2} \mathrm{~d} x \\
=\sum_{j=0}^{(n+1) / 2}\left(\begin{array}{c}
\frac{n+1}{2} \\
j
\end{array}\right) \int_{|x| \leq R^{1 / 2}}\left|D^{j} f_{R}(x)\right|^{2} \mathrm{~d} x+e^{2 R} \sum_{j=0}^{(n+1) / 2}\left(\begin{array}{c}
\frac{n+1}{2} \\
j
\end{array}\right) \int_{|x| \geq R^{1 / 2}}\left|D^{j} f_{0}(x)\right|^{2} \mathrm{~d} x
\end{gathered}
$$




$$
=I+I I
$$

We can estimate $I$ by the term corresponding to $j=(n+1) / 2$, that is,

$$
I \lesssim R^{-((n+1) / 2-1)} R^{n / 2}=R^{1 / 2},
$$

and by the dominated convergence theorem, $I I$ tends to $\left\|f_{0}\right\|_{H^{(n+1) / 2}\left(\mathbb{R}^{n}\right)}^{2}$ as $R \rightarrow 0$. Therefore

$$
\left\|f_{R}\right\|_{H^{(n+1) / 2}}^{2} \rightarrow\left\|f_{0}\right\|_{H^{(n+1) / 2}}^{2}=n ! \omega_{n}
$$

as $R \rightarrow 0$. This shows that $|B(0, R)| \rightarrow 1$ as $R \rightarrow 0$.

Since for any nonempty compact set $X$ we have (after suitable translation) $\{0\} \subseteq$ $R X \subseteq B(0, R M)$ for some $M>0$, we immediately deduce that $|R X| \rightarrow 1$ as $R \rightarrow 0$. (We thank Mark Meckes for pointing out this last implication to us.)

\section{Preliminaries on Sobolev spaces}

Let $\Omega$ be an arbitrary open set in $\mathbb{R}^{n}$. We need to consider some variants of the Sobolev spaces $H^{m}(\Omega)$ consisting of complex-valued functions whose weak derivatives of order up to and including $m \in \mathbb{N}$ belong to $L^{2}(\Omega)$. We shall assume the standard properties of $H^{m}\left(\mathbb{R}^{n}\right)$ and $H^{m}(\Omega)$ without specific mention. See [5] or [18] for more details.

Firstly, the space $H_{0}^{m}(\Omega)$ is the completion of $C_{c}^{\infty}(\Omega)$, the class of smooth functions of compact support inside $\Omega$, under the inner product

$$
\langle f, g\rangle_{H^{m}(\Omega)}:=\sum_{j=0}^{m}\left(\begin{array}{c}
m \\
j
\end{array}\right) \int_{\Omega} D^{j} f \cdot \overline{D^{j} g}
$$

where $D^{j} f=\Delta^{j / 2} f$ for $j$ even and $D^{j} f=\nabla \Delta^{(j-1) / 2} f$ for $j$ odd. Here and throughout

$$
\nabla f:=\left(\frac{\partial f}{\partial x_{1}}, \ldots, \frac{\partial f}{\partial x_{n}}\right) \text { and } \Delta f=\sum_{i=1}^{n} \frac{\partial^{2} f}{\partial x_{i}^{2}} .
$$

The inner product $\langle\cdot, \cdot\rangle_{H^{m}}$ gives rise to the norm $\|\cdot\|_{H^{m}}$ and when $\Omega=\mathbb{R}^{n}$ we can easily see using the Fourier transform that

$$
\|f\|_{H^{m}\left(\mathbb{R}^{n}\right)}^{2}=\left\|(I-\Delta)^{m / 2} f\right\|_{L^{2}\left(\mathbb{R}^{n}\right)}^{2} .
$$

We shall be working from now on with real-valued functions, and so we shall suppress the complex conjugate occurring in (10).

Let $K \subseteq \mathbb{R}^{n}$ be compact and convex with nonempty interior. If $f \in H^{m}\left(\mathbb{R}^{n}\right)$ and $g \in H^{m}(\operatorname{int} K)$ we say that $f=g$ on $K$ if $\|f-g\|_{H^{m}(\operatorname{int} K)}=0$. If $f=g$ on $K$ in this sense, then clearly $f=g$ almost everywhere on int $K$; conversely if $f \in H^{m}\left(\mathbb{R}^{n}\right)$, $g \in H^{m}$ (int $K$ ) and $f=g$ almost everywhere on int $\mathrm{K}$, then the weak derivatives of $f$ and $g$ of order up to and including $m$ on int K coincide, and so $f=g$ on $K$. If $m>n / 2$ the Sobolev embedding theorem implies that the functions in $H^{m}\left(\mathbb{R}^{n}\right)$ are continuous and hence make sense pointwise on $K$; so the statement $f=g$ on $K$ can be interpreted pointwise in this case.

With this in mind, for our second variant we define

$$
\tilde{H}_{0}^{m}\left(\mathbb{R}^{n} \backslash K\right):=\left\{f \in H^{m}\left(\mathbb{R}^{n}\right): f=0 \text { on } K\right\} .
$$


This is clearly a closed subspace of $H^{m}\left(\mathbb{R}^{n}\right)$, and there are natural isometric embeddings

$$
H_{0}^{m}\left(\mathbb{R}^{n} \backslash K\right) \hookrightarrow \tilde{H}_{0}^{m}\left(\mathbb{R}^{n} \backslash K\right) \hookrightarrow H^{m}\left(\mathbb{R}^{n}\right) .
$$

Under our hypothesis that the interior of $K$ is nonempty, the first embedding is surjective:

Lemma 1. If $K$ has nonempty interior then for any $f \in H^{m}\left(\mathbb{R}^{n}\right)$ such that $f=0$ on $K$, and any $\epsilon>0$, there is a $\phi \in C_{c}^{\infty}\left(\mathbb{R}^{n} \backslash K\right)$ such that $\|f-\phi\|_{H^{m}\left(\mathbb{R}^{n}\right)}<\epsilon$.

Proof. See for example Theorem 5.29 of [2].

We need some more lemmas.

Lemma 2. If $f$ is in $H^{m}\left(\mathbb{R}^{n}\right), K$ is any compact subset of $\mathbb{R}^{n}$ and $\epsilon>0$ we may choose a smooth cut-off function $\psi$ which is identically 1 on $K$ and is such that if $\tilde{f}=\psi f$, then $\|f-\tilde{f}\|_{H^{m}\left(\mathbb{R}^{n}\right)}<\epsilon$.

Proof. If $\Phi$ is a standard normalised bump function which is identically one on the unit ball, and zero off the ball of radius 2 we have

$$
\|f(1-\Phi(\cdot / R))\|_{H^{m}\left(\mathbb{R}^{n}\right)} \leq C \sum_{j=0}^{m}\left(\begin{array}{c}
m \\
j
\end{array}\right) \int_{|x| \geq R}\left|D^{j} f\right|^{2}
$$

and for $f$ in $H^{m}\left(\mathbb{R}^{n}\right)$ we have

$$
\sum_{j=0}^{m}\left(\begin{array}{c}
m \\
j
\end{array}\right) \int_{|x| \geq R}\left|D^{j} f\right|^{2} \rightarrow 0 \text { as } R \rightarrow \infty .
$$

Lemma 3. Suppose that $f \in H^{m}\left(\mathbb{R}^{n}\right)$ and that $f=1$ on $K$ where $K$ has nonempty interior. Suppose that $\psi$ is a smooth cut-off function which is identically 1 on a neighbourhood of $K$. Let $\tilde{f}(x)=(f(x)-1) \psi(x)$. Then $\tilde{f} \in H_{0}^{m}\left(\mathbb{R}^{n} \backslash K\right)$.

Proof. Clearly $\tilde{f}$ belongs to $H^{m}\left(\mathbb{R}^{n}\right)$ with $\|\tilde{f}\|_{H^{m}\left(\mathbb{R}^{n}\right)} \leq C\left(\|f\|_{H^{m}\left(\mathbb{R}^{n}\right)}+1\right)$ where $C$ depends on $\psi$ and $K$. Moreover $\tilde{f}=0$ on $K$. So by Lemma $1 \tilde{f}$ belongs to $H_{0}^{m}\left(\mathbb{R}^{n} \backslash K\right)$ with the same control.

For convenience we also recall the facts about traces we shall need from 2 or 5 . (See also 1.).) In the lemma which follows, $\mathcal{D}^{j}$ denotes any differential operator of order $j$ with constant coefficients. (We shall apply it in the special case that $\mathcal{D}^{j}=\Delta^{j / 2}$ for $j$ even and $\mathcal{D}^{j}=(\nu \cdot \nabla) \Delta^{(j-1) / 2}$ for $j$ odd, where $\nu$ is some unit vector.)

Lemma 4. Suppose $f \in H^{m}\left(\mathbb{R}^{n}\right)$ and that $1 \leq j \leq m-1$. Then

(i) If $S$ is a piecewise smooth compact hypersurface, then $\mathcal{D}^{j} f$ belongs to $H^{m-j-1 / 2}(S)$ and

$$
\left\|\left.\mathcal{D}^{j} f\right|_{S}\right\|_{H^{m-j-1 / 2}(S)} \leq C\|f\|_{H^{m}\left(\mathbb{R}^{n}\right)}
$$

moreover the map $\left.f \mapsto \mathcal{D}^{j} f\right|_{S}$ varies continuously with small changes in $S$.

(ii) If $K$ is a compact convex set in $\mathbb{R}^{n}$ with nonempty interior and $f \in H_{0}^{m}\left(\mathbb{R}^{n} \backslash\right.$ $K)$, then $\left.\mathcal{D}^{j} f\right|_{\partial K}=0$. 
Proof. (i) Since $f \in H^{m}\left(\mathbb{R}^{n}\right)$ we have $\mathcal{D}^{j} f \in H^{m-j}\left(\mathbb{R}^{n}\right)$ (with norm control) and so by the classical trace inequality its restriction to the hypersurface $S$ is in $H^{m-j-1 / 2}(S)$ provided $m-j \geq 1$, again with norm control. To see the continuity, suppose $S$ and $S^{\prime}$ are nearby hypersurfaces, and take $\phi \in C_{c}^{\infty}\left(\mathbb{R}^{n}\right)$ and $\epsilon>0$ such that $\|f-\phi\|_{H^{m}\left(\mathbb{R}^{n}\right)}<\epsilon$. Then

$$
\begin{gathered}
\left\|\left.\mathcal{D}^{j} f\right|_{S}-\left.\mathcal{D}^{j} f\right|_{S^{\prime}}\right\|_{H^{m-j-1 / 2}} \\
\leq\left\|\left.\left(\mathcal{D}^{j} f-\mathcal{D}^{j} \phi\right)\right|_{S}\right\|_{H^{m-j-1 / 2}}+\left\|\left.\mathcal{D}^{j} \phi\right|_{S}-\left.\mathcal{D}^{j} \phi\right|_{S^{\prime}}\right\|_{H^{m-j-1 / 2}}+\left\|\left.\left(\mathcal{D}^{j} \phi-\mathcal{D}^{j}\right) f\right|_{S^{\prime}}\right\|_{H^{m-j-1 / 2}} .
\end{gathered}
$$

The first and third terms are dominated by $\epsilon$ by the first part and the second term goes to zero as $S^{\prime} \rightarrow S$ since $\phi$ is smooth.

(ii) This is a consequence of the classical characterisation of the traces of functions in the Sobolev spaces $H_{0}^{m}\left(\mathbb{R}^{n} \backslash K\right)$. Alternatively, it follows from the previous part since for smooth hypersurfaces contained in the interior of $K$ we will have $\left.\mathcal{D}^{j} f\right|_{S}=0$ for all $j \geq 1$.

\section{The VARiational PROBlem FOR THE EXTREMAL ENERGY}

In this section we consider the variational problem

$$
\inf \left\{\|f\|_{H^{m}\left(\mathbb{R}^{n}\right)}: f \in H^{m}\left(\mathbb{R}^{n}\right), f=g \text { on } K\right\}
$$

where $g$ is a prescribed member of $H^{m}\left(\mathbb{R}^{n}\right), m \in \mathbb{N}$ and $K$ is a compact convex set in $\mathbb{R}^{n}$ with nonempty interior. We call this problem the $m$-extremal energy problem for $K$, or simply the extremal energy problem.

Proposition 1. The extremal energy problem

$$
\inf \left\{\|f\|_{H^{m}\left(\mathbb{R}^{n}\right)}: f \in H^{m}\left(\mathbb{R}^{n}\right), f=g \text { on } \mathrm{K}\right\}
$$

has a unique solution.

Proof. This is just the elementary fact that in the Hilbert space $H^{m}\left(\mathbb{R}^{n}\right)$, there is a unique closest point to zero in the nonempty closed convex (in fact affine) set $\left\{\|f\|_{H^{m}\left(\mathbb{R}^{n}\right)}: f \in H^{m}\left(\mathbb{R}^{n}\right), f=g\right.$ on $\left.K\right\}$.

When $K$ is a Euclidean ball we can see immediately that the unique solution to the extremal energy problem is radial since averaging any solution over rotations yields another solution which is radial.

By standard arguments (see for example [17] for the case $m=(n+1) / 2$ ), the Euler-Lagrange equation for the extremal energy problem is

$$
(I-\Delta)^{m} f=0 \text { on } \mathbb{R}^{n} \backslash K
$$

in the weak sense (testing against functions in $C_{c}^{\infty}\left(\mathbb{R}^{n} \backslash K\right)$ ) and so we are led to study the problem

$$
\begin{aligned}
(I-\Delta)^{m} f & =0 \text { on } \mathbb{R}^{n} \backslash K \text { in the weak sense } \\
f & =g \text { on } K .
\end{aligned}
$$

We immediately have existence of solutions in $H^{m}\left(\mathbb{R}^{n}\right)$ to this problem from Proposition [1, and we also have uniqueness: 
Proposition 2. Suppose that $g \in H^{m}\left(\mathbb{R}^{n}\right)$. Then there is a unique solution to the problem

$$
\begin{aligned}
(I-\Delta)^{m} f & =0 \text { weakly on } \mathbb{R}^{n} \backslash K \\
f & =g \text { on } K
\end{aligned}
$$

with $f \in H^{m}\left(\mathbb{R}^{n}\right)$.

Proof. The space of solutions to (11) is $f_{0}+\mathcal{M}$ where $f_{0}$ is any particular solution and $\mathcal{M}$ is the orthogonal complement of $H_{0}^{m}\left(\mathbb{R}^{n} \backslash K\right)$ in $\tilde{H}_{0}^{m}\left(\mathbb{R}^{n} \backslash K\right)$. But by Lemma $1 \mathcal{M}=\{0\}$, and so the solution is unique.

So the unique solution to the extremal energy problem of Proposition 1 is also the unique solution to (11) in $H^{m}\left(\mathbb{R}^{n}\right)$.

We shall need in the next section to appeal to the theory of elliptic regularity. Briefly, for an elliptic operator such as $(I-\Delta)^{m}$, weak solutions $f$ to $(I-\Delta)^{m} f=0$ on an open set are actually smooth, and the equation $(I-\Delta)^{m} f=0$ holds in the classical sense. See [2] or [5].

\section{A fORMUla FOR THE $m$-EXTREMAL ENERGy}

In this section we develop the promised formula for the $m$-extremal energy. See Theorem 5 below.

We first consider arbitrary solutions $h \in H^{m}\left(\mathbb{R}^{n}\right)$ of the problem

$$
\left\{\begin{array}{rl}
(I-\Delta)^{m} & h=0 \text { weakly on } \mathbb{R}^{n} \backslash K \\
h & =1 \text { on } K
\end{array}\right.
$$

where $m \geq 1$ is an arbitrary integer and $K$ is a compact convex set with nonempty interior. Consider a regularised distance function $d$ for $K$, which is defined on $\mathbb{R}^{n}$ and satisfies $d(x) \sim \operatorname{dist}(x, K)$. Let $K_{r}=\left\{x \in \mathbb{R}^{n}: d(x) \leq r\right\}$ and let $\nu$ denote the unit normal pointing out of $K_{r}$. (Note that $\partial K_{r}$ is smooth and so Lemma 4 will be applicable.)

For $g \in H^{m}\left(\mathbb{R}^{n}\right)$ with compact support we have

$$
\begin{aligned}
\langle g, h\rangle_{H^{m}\left(\mathbb{R}^{n}\right)} & =\sum_{j=0}^{m}\left(\begin{array}{c}
m \\
j
\end{array}\right) \int_{\mathbb{R}^{n}} D^{j} g \cdot D^{j} h \\
& =\int_{K} g+\sum_{j=0}^{m}\left(\begin{array}{c}
m \\
j
\end{array}\right) \lim _{r \downarrow 0} \int_{\{d(x) \geq r\}} D^{j} g \cdot D^{j} h
\end{aligned}
$$

by the dominated convergence theorem.

We shall study the terms $\int_{\{d(x) \geq r\}} D^{j} g \cdot D^{j} h$ by integrating by parts. In so doing, we shall systematically use Green's formulae

$$
\begin{aligned}
\int_{\Omega} \nabla \phi \cdot \nabla \psi & =-\int_{\Omega} \phi \Delta \psi-\int_{\partial \Omega} \phi \frac{\partial \psi}{\partial \nu} \mathrm{d} S \\
\text { and } \int_{\Omega}(\Delta \phi) \psi & =-\int_{\Omega} \nabla \phi \cdot \nabla \psi-\int_{\partial \Omega} \frac{\partial \phi}{\partial \nu} \psi \mathrm{d} S
\end{aligned}
$$


(where we are trying to increase the differentiability of $\psi$, which will be a function of $h$, with the eventual aim of using the equation satisfied by $h$, and decrease that of $\phi$, which will be a function of $g$ ). In these formulae the region $\Omega$ will be $\{d(x) \geq r\}$ and $\nu$ is the unit normal pointing out of $K_{r}$. The smoothness of $h$ on $\mathbb{R}^{n} \backslash K$ coming from elliptic regularity, and the compact support of $g$ will ensure that each use of these formulae is valid. In particular the compact support of $g$ means that there are no boundary terms at infinity to consider.

To carry out these calculations it is convenient to define $\mathcal{D}^{j} f:=\Delta^{j / 2} f$ for $j$ even and $\mathcal{D}^{j} f:=\frac{\partial}{\partial \nu} \Delta^{(j-1) / 2} f$ for $j$ odd. (Note the distinction here when $j$ is odd between $\mathcal{D}$ and $D$. While a risking a possible typographical confusion, at a first reading one should simply think of $\mathcal{D}^{j}$ and $D^{j}$ as denoting suitable derivatives of order $j$. Note also that these boundary operators occur in the PDE literature in problems involving hybrid Dirichlet-Navier boundary conditions. See for example [6], p.33. It is in the lemma below that expressions such as $\Delta u$ (rather than $\frac{\partial^{2} u}{\partial \nu^{2}}$ ) appear more naturally on the boundary.

Lemma 5. Let $K \subseteq \mathbb{R}^{n}$ be compact and convex. For $1 \leq j \leq m$ and $g \in H^{m}\left(\mathbb{R}^{n}\right)$ with compact support we have

$$
\int_{\{d(x) \geq r\}} D^{j} g \cdot D^{j} h=(-1)^{j}\left(\int_{\{d(x) \geq r\}} g \Delta^{j} h+\sum_{k=0}^{j-1}(-1)^{k} \int_{\partial K_{r}} \mathcal{D}^{k} g \mathcal{D}^{2 j-k-1} h \mathrm{~d} S\right) .
$$

Proof. Let us first consider the case $j=1$. Then

$$
\begin{aligned}
\int_{\{d(x) \geq r\}} \nabla g \cdot \nabla h & =-\int_{\{d(x) \geq r\}} g \Delta h-\int_{\partial K_{r}} g \frac{\partial h}{\partial \nu} \mathrm{d} S \\
& =-\left(\int_{\{d(x) \geq r\}} g \Delta h+\int_{\partial K_{r}} g \mathcal{D} h \mathrm{~d} S\right)
\end{aligned}
$$

as required.

Now, for $j=2$ we have

$$
\begin{aligned}
\int_{\{d(x) \geq r\}}^{\Delta g \Delta h} & =-\int_{\{d(x) \geq r\}} \nabla g \cdot \nabla \Delta h-\int_{\partial K_{r}} \frac{\partial g}{\partial \nu} \Delta h \mathrm{~d} S \\
& =\int_{\{d(x) \geq r\}} g \Delta^{2} h+\int_{\partial K_{r}} g \frac{\partial \Delta h}{\partial \nu} \mathrm{d} S-\int_{\partial K_{r}} \frac{\partial g}{\partial \nu} \Delta h \mathrm{~d} S \\
& =\int_{\{d(x) \geq r\}} g \Delta^{2} h+\int_{\partial K_{r}} g \mathcal{D}^{3} h \mathrm{~d} S-\int_{\partial K_{r}} \mathcal{D} g \mathcal{D}^{2} h \mathrm{~d} S
\end{aligned}
$$

as required, noting that the term $\frac{\partial g}{\partial \nu}$ makes sense as a member of $H^{m-3 / 2}$ of the hypersurface $\partial K_{r}$ by the trace inequality, Lemma 4. (For this case to occur we must have $m \geq 2$.) 
Similarly, for $j=3$, we have

$$
\begin{aligned}
& \int_{\{d(x) \geq r\}} \nabla \Delta g \cdot \nabla \Delta h \\
& =-\int_{\{d(x) \geq r\}} \Delta g \Delta^{2} h-\int_{\partial K_{r}} \Delta g \frac{\partial \Delta h}{\partial \nu} \mathrm{d} S \\
& =\int_{\{d(x) \geq r\}} \nabla g \cdot \nabla \Delta^{2} h+\int_{\partial K_{r}} \frac{\partial g}{\partial \nu} \Delta^{2} h \mathrm{~d} S-\int_{\partial K_{r}} \Delta g \frac{\partial \Delta h}{\partial \nu} \mathrm{d} S \\
& =-\int_{\{d(x) \geq r\}} g \Delta^{3} h-\int_{\partial K_{r}} g \frac{\partial \Delta^{2} h}{\partial \nu} \mathrm{d} S+\int_{\partial K_{r}} \frac{\partial g}{\partial \nu} \Delta^{2} h \mathrm{~d} S-\int_{\partial K_{r}} \Delta g \frac{\partial \Delta h}{\partial \nu} \mathrm{d} S \\
& =-\left(\int_{\{d(x) \geq r\}} g \Delta^{3} h+\int_{\partial K_{r}} g \mathcal{D}^{5} h \mathrm{~d} S-\int_{\partial K_{r}} \mathcal{D} g \mathcal{D}^{4} h \mathrm{~d} S+\int_{\partial K_{r}} \mathcal{D}^{2} g \mathcal{D}^{3} h \mathrm{~d} S\right)
\end{aligned}
$$

noting that the terms $\frac{\partial g}{\partial \nu}$ and $\Delta g$ make sense as members of $H^{m-3 / 2}$ and $H^{m-5 / 2}$ respectively of the hypersurface $\partial K_{r}$ by the trace inequality, Lemma 4 . (For this case to occur we must have $m \geq 3$.)

Continuing, we see that the general case takes the form

$$
\begin{gathered}
\int_{\{d(x) \geq r\}} D^{j} g \cdot D^{j} h \\
=(-1)^{j}\left\{\int_{\{d(x) \geq r\}} g \Delta^{j} h+\int_{\partial K_{r}} g \frac{\partial \Delta^{j-1} h}{\partial \nu} \mathrm{d} S-\int_{\partial K_{r}} \frac{\partial g}{\partial \nu} \Delta^{j-1} h \mathrm{~d} S\right. \\
\left.+\int_{\partial K_{r}} \Delta g \frac{\partial \Delta^{j-2} h}{\partial \nu} \mathrm{d} S-\int_{\partial K_{r}} \frac{\partial \Delta g}{\partial \nu} \Delta^{j-2} h \mathrm{~d} S+\ldots\right\}
\end{gathered}
$$

where the last term inside the curly brackets is

$$
-\int_{\partial K_{r}} \frac{\partial \Delta^{(j-2) / 2} g}{\partial \nu} \Delta^{j / 2} h \mathrm{~d} S
$$

when $j$ is even and

$$
+\int_{\partial K_{r}} \Delta^{(j-1) / 2} g \frac{\partial \Delta^{(j-1) / 2} h}{\partial \nu} \mathrm{d} S
$$

when $j$ is odd. That is,

$$
\begin{gathered}
\int_{\{d(x) \geq r\}} D^{j} h \cdot D^{j} g \\
=(-1)^{j}\left\{\int_{\{d(x) \geq r\}} g \Delta^{j} h+\int_{\partial K_{r}} g \mathcal{D}^{(2 j-1)} h \mathrm{~d} S-\int_{\partial K_{r}} \mathcal{D} g \mathcal{D}^{(2 j-2)} h \mathrm{~d} S\right. \\
+\int_{\partial K_{r}} \mathcal{D}^{2} g \mathcal{D}^{(2 j-3)} h \mathrm{~d} S-\int_{\partial K_{r}} \mathcal{D}^{3} g \mathcal{D}^{(2 j-4)} h \mathrm{~d} S \\
\left.+\cdots+(-1)^{j-1} \int_{\partial K_{r}} \mathcal{D}^{(j-1)} g \mathcal{D}^{j} h \mathrm{~d} S\right\} .
\end{gathered}
$$

Note that the terms $\mathcal{D} g, \mathcal{D}^{2} g, \ldots, \mathcal{D}^{(j-1)} g$ make sense as members of $H^{m-3 / 2}$, $H^{m-5 / 2}, \ldots, H^{m-j+1 / 2}$ respectively of the hypersurface $\partial K_{r}$ by the trace inequality, Lemma 4. (For this case to occur we must have $m \geq j$.) This establishes the lemma. 
If we take the identities of Lemma [5 multiply by $\left(\begin{array}{c}m \\ j\end{array}\right)$ and sum from $j=1$ to $m$ we get

$$
\begin{aligned}
& \sum_{j=1}^{m}\left(\begin{array}{c}
m \\
j
\end{array}\right) \int_{\{d(x) \geq r\}} D^{j} g \cdot D^{j} h \\
= & \sum_{j=1}^{m}\left(\begin{array}{c}
m \\
j
\end{array}\right)(-1)^{j}\left(\int_{\{d(x) \geq r\}} g \Delta^{j} h+\sum_{k=0}^{j-1}(-1)^{k} \int_{\partial K_{r}} \mathcal{D}^{k} g \mathcal{D}^{2 j-k-1} h \mathrm{~d} S\right) \\
= & \int_{\{d(x) \geq r\}} g\left(\sum_{j=1}^{m}\left(\begin{array}{c}
m \\
j
\end{array}\right)(-1)^{j} \Delta^{j} h\right)+\sum_{j=1}^{m}\left(\begin{array}{c}
m \\
j
\end{array}\right)(-1)^{j} \sum_{k=0}^{j-1}(-1)^{k} \int_{\partial K_{r}} \mathcal{D}^{k} g \mathcal{D}^{2 j-k-1} h \mathrm{~d} S .
\end{aligned}
$$

Now add $\int_{\{d(x) \geq r\}} g h$ (corresponding to the the term $j=0$ ) to both sides to obtain

$$
\begin{aligned}
& \sum_{j=0}^{m}\left(\begin{array}{c}
m \\
j
\end{array}\right) \int_{\{d(x) \geq r\}} D^{j} h \cdot D^{j} g \\
= & \int_{\{d(x) \geq r\}} g\left(\sum_{j=0}^{m}\left(\begin{array}{c}
m \\
j
\end{array}\right)(-1)^{j} \Delta^{j} h\right)+\sum_{0 \leq k<j \leq m}(-1)^{j+k}\left(\begin{array}{c}
m \\
j
\end{array}\right) \int_{\partial K_{r}} \mathcal{D}^{k} g \mathcal{D}^{2 j-k-1} h \mathrm{~d} S \\
= & \int_{\{d(x) \geq r\}} g(I-\Delta)^{m} h+\sum_{0 \leq k<j \leq m}(-1)^{j+k}\left(\begin{array}{c}
m \\
j
\end{array}\right) \int_{\partial K_{r}} \mathcal{D}^{k} g \mathcal{D}^{2 j-k-1} h \mathrm{~d} S \\
= & \sum_{0 \leq k<j \leq m}(-1)^{j+k}\left(\begin{array}{c}
m \\
j
\end{array}\right) \int_{\partial K_{r}} \mathcal{D}^{k} g \mathcal{D}^{2 j-k-1} h \mathrm{~d} S
\end{aligned}
$$

since $h$ satisfies the equation $(I-\Delta)^{m} h=0$ in the classical sense on $\{d(x)>0\}$ by elliptic regularity.

Combining this with (12), we obtain the representation

$$
\langle g, h\rangle_{H^{m}\left(\mathbb{R}^{n}\right)}=\int_{K} g+\lim _{r \downarrow 0} \sum_{0 \leq k<j \leq m}(-1)^{j+k}\left(\begin{array}{c}
m \\
j
\end{array}\right) \int_{\partial K_{r}} \mathcal{D}^{k} g \mathcal{D}^{2 j-k-1} h \mathrm{~d} S
$$

valid for any $g \in H^{m}\left(\mathbb{R}^{n}\right)$ with compact support.

We would like to extend this formula to be valid for all $g \in H^{m}\left(\mathbb{R}^{n}\right)$. Observe that all the terms on the right hand side make sense for such $g$ - as was noted during the proof of Lemma 5. In particular there is no problem with the existence of the limit as $r \downarrow 0$ for such $g$ - simply multiply it by a smooth cut-off which is identically 1 on $\{d(x) \leq 2\}$ to obtain $\tilde{g}$ of compact support for which the corresponding limit exists and the value of which is left unaltered by the multiplication.

So the linear functional $\Lambda$ given by

$$
\Lambda g:=\langle g, h\rangle_{H^{m}\left(\mathbb{R}^{n}\right)}-\int_{K} g-\lim _{r \downarrow 0}\left(\sum_{0 \leq k<j \leq m}(-1)^{j+k}\left(\begin{array}{c}
m \\
j
\end{array}\right) \int_{\{d(x)=r\}} \mathcal{D}^{k} g \mathcal{D}^{2 j-k-1} h \mathrm{~d} S\right)
$$

is well-defined on $H^{m}\left(\mathbb{R}^{n}\right)$ and is identically zero on the dense subspace consisting of functions of compact support. If $g$ is in $H^{m}\left(\mathbb{R}^{n}\right)$ and $\epsilon>0$ we may choose by 
Lemma 2 a cut-off function $\psi$ which is identically 1 on $\{d(x) \leq 2\}$ and such that if $\tilde{g}=\psi g$, then $\|g-\tilde{g}\|_{H^{m}\left(\mathbb{R}^{n}\right)}<\epsilon$. Hence

$$
\Lambda g=\Lambda g-\Lambda \tilde{g}=\Lambda(g-\tilde{g})=\langle g-\tilde{g}, h\rangle_{H^{m}\left(\mathbb{R}^{n}\right)}
$$

so that

$$
|\Lambda g| \leq\|g-\tilde{g}\|_{H^{m}\left(\mathbb{R}^{n}\right)}\|h\|_{H^{m}\left(\mathbb{R}^{n}\right)}<\epsilon\|h\|_{H^{m}\left(\mathbb{R}^{n}\right)} .
$$

Thus $\Lambda g=0$ for all $g \in H^{m}\left(\mathbb{R}^{n}\right)$ and we have:

Proposition 3. For $m \in \mathbb{N}, K$ any compact convex set in $\mathbb{R}^{n}$ and any solution $h \in H^{m}\left(\mathbb{R}^{n}\right)$ to the problem $(I-\Delta)^{m} h=0$ weakly on $\mathbb{R}^{n} \backslash K$, we have the formula

$$
\langle g, h\rangle_{H^{m}\left(\mathbb{R}^{n}\right)}=\int_{K} g+\lim _{r \downarrow 0} \sum_{0 \leq k<j \leq m}(-1)^{j+k}\left(\begin{array}{c}
m \\
j
\end{array}\right) \int_{\partial K_{r}} \mathcal{D}^{k} g \mathcal{D}^{2 j-k-1} h \mathrm{~d} S,
$$

valid for every $g \in H^{m}\left(\mathbb{R}^{n}\right)$.

It is intuitively reasonable that for functions $g \in H_{0}^{m}\left(\mathbb{R}^{n} \backslash K\right)$ there should be no boundary terms in formula (13) and indeed for such $g$ it is natural that $\int_{K} g=0$ and also that $\langle g, h\rangle_{H^{m}}=0$ using the equation. This we establish next. (Note that in the following lemma we had better use the equation satisfied by $h$ as otherwise there is no reason to believe the terms involving higher derivatives of $h$ exist.)

Lemma 6. For $g \in H_{0}^{m}\left(\mathbb{R}^{n} \backslash K\right)$ and $h \in H^{m}\left(\mathbb{R}^{n}\right)$ any solution to $(I-\Delta)^{m} h=0$ weakly on $\mathbb{R}^{n} \backslash K$ we have

$$
\lim _{r \downarrow 0} \sum_{0 \leq k<j \leq m}(-1)^{j+k}\left(\begin{array}{c}
m \\
j
\end{array}\right) \int_{\partial K_{r}} \mathcal{D}^{k} g \mathcal{D}^{2 j-k-1} h \mathrm{~d} S=0 .
$$

On the other hand, if $g \in H^{m}\left(\mathbb{R}^{n}\right)$, if $g=0$ on $K$ (i.e. $g=0$ pointwise on $K$ if $m>n / 2$, and is zero on int $K \neq \emptyset$ when $m \leq n / 2)$ and if

$$
\lim _{r \downarrow 0} \sum_{0 \leq k<j \leq m}(-1)^{j+k}\left(\begin{array}{c}
m \\
j
\end{array}\right) \int_{\partial K_{r}} \mathcal{D}^{k} g \mathcal{D}^{2 j-k-1} h \mathrm{~d} S=0
$$

for every solution $h \in H^{m}\left(\mathbb{R}^{n}\right)$ to $(I-\Delta)^{m} h=0$ weakly on $\mathbb{R}^{n} \backslash K$ which also satisfies $h=0$ on $K$, then $g \in H_{0}^{m}\left(\mathbb{R}^{n} \backslash K\right)$.

Proof. Consider the first assertion. By our formula (13) we have

$$
\lim _{r \downarrow 0} \sum_{0 \leq k<j \leq m}(-1)^{j+k}\left(\begin{array}{c}
m \\
j
\end{array}\right) \int_{\partial K_{r}} \mathcal{D}^{k} g \mathcal{D}^{2 j-k-1} h \mathrm{~d} S=\langle g, h\rangle_{H^{m}\left(\mathbb{R}^{n}\right)}-\int_{K} g .
$$

So it is enough to show that

$$
\Lambda g:=\langle g, h\rangle_{H^{m}\left(\mathbb{R}^{n}\right)}-\int_{K} g
$$

satisfies

$$
\Lambda g=0
$$

for all $g \in H_{0}^{m}\left(\mathbb{R}^{n} \backslash K\right)$. Let $\epsilon>0$ and $\tilde{g} \in C_{c}^{\infty}\left(\mathbb{R}^{n} \backslash K\right)$ be such that $\| g-$ $\tilde{g} \|_{H_{0}^{m}\left(\mathbb{R}^{n} \backslash K\right)}<\epsilon$. Clearly we have $\Lambda \tilde{g}=0$ since $h$ satisfies $(I-\Delta)^{m} h=0$ weakly on $\mathbb{R}^{n} \backslash K$ and $K \cap \mathbb{R}^{n} \backslash K=\emptyset$, and so

$$
\Lambda g=\Lambda g-\Lambda \tilde{g}=\Lambda(g-\tilde{g})=\langle g-\tilde{g}, h\rangle_{H^{m}\left(\mathbb{R}^{n}\right)}-\int_{K}(g-\tilde{g}) h,
$$


thus

$$
|\Lambda g| \leq\|g-\tilde{g}\|_{H^{m}\left(\mathbb{R}^{n}\right)}\|h\|_{H^{m}\left(\mathbb{R}^{n}\right)}+\|g-\tilde{g}\|_{2}\|h\|_{2}<\epsilon\|h\|_{H^{m}\left(\mathbb{R}^{n}\right)} .
$$

Hence $\Lambda g=0$ for all $g \in H_{0}^{m}\left(\mathbb{R}^{n} \backslash K\right)$.

For the second assertion, if the limit in question is zero, we have by the representation formula (13) that $\langle g, h\rangle_{H^{m}}=0$ for all $h \in H^{m}\left(\mathbb{R}^{n}\right)$ which are solutions to $(I-\Delta)^{m} h=0$ weakly on $\mathbb{R}^{n} \backslash K$ which also satisfy $h=0$ on $K$. So $g \in \tilde{H}_{0}^{m}\left(\mathbb{R}^{n} \backslash K\right)$ and $g$ is perpendicular to the space $\mathcal{M}$ introduced above in Proposition 2 . By definition of $\mathcal{M}$ this means that $g \in H_{0}^{m}\left(\mathbb{R}^{n} \backslash K\right)$.

We have not yet used the requirement that $K$ have nonempty interior nor used any boundary conditions satisfied by $h$ (except to obtain the formula (12)). In order to deduce a useful formula for $\|h\|_{H^{m}\left(\mathbb{R}^{n}\right)}^{2}$, we shall have to do so, and we shall use Lemma 3 freely from now on.

So, let $H$ be any function in $H^{m}\left(\mathbb{R}^{n}\right)$ such that $H=1$ on $K$. Then, by Lemma 3 we have that $g(x)=(H(x)-1) \psi(x)$ belongs to $H_{0}^{m}\left(\mathbb{R}^{n} \backslash K\right.$ ) (where $\psi$ is any smooth function of compact support which is identically 1 on $\{d(x) \leq 2\})$, and so we can apply Lemma 6 to obtain

$$
\begin{aligned}
& \lim _{r \downarrow 0} \sum_{0 \leq k<j \leq m}(-1)^{j+k}\left(\begin{array}{c}
m \\
j
\end{array}\right) \int_{\partial K_{r}} \mathcal{D}^{k} H \mathcal{D}^{2 j-k-1} h \mathrm{~d} S \\
- & \lim _{r \downarrow 0} \sum_{0 \leq k<j \leq m}(-1)^{j+k}\left(\begin{array}{c}
m \\
j
\end{array}\right) \int_{\partial K_{r}} \mathcal{D}^{k} \psi \mathcal{D}^{2 j-k-1} h \mathrm{~d} S=0 .
\end{aligned}
$$

But for $r$ sufficiently close to zero we have

$$
\begin{aligned}
& \sum_{0 \leq k<j \leq m}(-1)^{j+k}\left(\begin{array}{c}
m \\
j
\end{array}\right) \int_{\partial K_{r}} \mathcal{D}^{k} \psi \mathcal{D}^{2 j-k-1} h \mathrm{~d} S \\
= & \sum_{j=1}^{m}\left(\begin{array}{c}
m \\
j
\end{array}\right)(-1)^{j} \int_{\partial K_{r}} \mathcal{D}^{2 j-1} h \mathrm{~d} S
\end{aligned}
$$

so that

$$
\begin{aligned}
& \lim _{r \downarrow 0} \sum_{0 \leq k<j \leq m}(-1)^{j+k}\left(\begin{array}{c}
m \\
j
\end{array}\right) \int_{\partial K_{r}} \mathcal{D}^{k} H \mathcal{D}^{2 j-k-1} h \mathrm{~d} S \\
= & \lim _{r \downarrow 0} \sum_{j=1}^{m}(-1)^{j}\left(\begin{array}{c}
m \\
j
\end{array}\right) \int_{\partial K_{r}} \mathcal{D}^{2 j-1} h \mathrm{~d} S .
\end{aligned}
$$

Next, we take $g=H$ in Proposition 3 and apply (14) to obtain:

Proposition 4. Suppose $K$ has nonempty interior, $h$ is any solution in $H^{m}\left(\mathbb{R}^{n} \backslash K\right)$ to $(I-\Delta)^{m} h=0$ weakly on $\mathbb{R}^{n} \backslash K$, and $H$ is any function in $H^{m}\left(\mathbb{R}^{n}\right)$ such that $H=1$ on $K$. Then we have

$$
\langle H, h\rangle_{H^{m}\left(\mathbb{R}^{n}\right)}=\operatorname{Vol}(K)+\lim _{r \downarrow 0} \sum_{j=1}^{m}(-1)^{j}\left(\begin{array}{c}
m \\
j
\end{array}\right) \int_{\partial K_{r}} \mathcal{D}^{2 j-1} h \mathrm{~d} S .
$$


Finally, we claim that the terms with $j \leq m / 2$ give a contribution of zero in this formula.

Indeed, if we have $h \in H^{m}\left(\mathbb{R}^{n}\right)$ and $h=1$ on $K$, Lemma 3 tells us that for suitable smooth $\psi$ of compact support, identically 1 on a neighbourhood of $K$ we have $g=(h-1) \psi \in H_{0}^{m}\left(\mathbb{R}^{n} \backslash K\right)$. So by Lemma $4 \mathcal{D}^{i} g$ is the zero member of $H^{m-i-1 / 2}(\partial K)$ for $0 \leq i \leq m-1$, and continuity of traces shows that we therefore have

$$
\int_{\partial K_{r}} \mathcal{D}^{i} g \mathrm{~d} S \rightarrow 0 \text { as } r \downarrow 0
$$

for $0 \leq i \leq m-1$.

In particular, since $\mathcal{D}^{i} h=\mathcal{D}^{i} g+\mathcal{D}^{i} \psi$ near $\partial K$ we have

$$
\int_{\partial K_{r}} \mathcal{D}^{i} h \mathrm{~d} S \rightarrow 0 \text { as } r \downarrow 0
$$

for $1 \leq i \leq m-1$. So

$$
\int_{\partial K_{r}} \mathcal{D}^{2 j-1} h \mathrm{~d} S \rightarrow 0 \text { as } r \downarrow 0
$$

for $1 \leq 2 j-1 \leq m-1$, that is for $1 \leq j \leq m / 2$.

Hence

$$
\langle H, h\rangle_{H^{m}\left(\mathbb{R}^{n}\right)}=\operatorname{Vol}(K)+\lim _{r \downarrow 0} \sum_{m / 2<j \leq m}(-1)^{j}\left(\begin{array}{c}
m \\
j
\end{array}\right) \int_{\partial K_{r}} \mathcal{D}^{2 j-1} h \mathrm{~d} S,
$$

and we have proved:

Theorem 5. Suppose that $K$ is a compact convex set in $\mathbb{R}^{n}$ which has nonempty interior. Let $H$ be any function in $H^{m}\left(\mathbb{R}^{n}\right)$ such that $H=1$ on $K$. Then the unique solution $h \in H^{m}\left(\mathbb{R}^{n}\right)$ to the problem

$$
\begin{gathered}
(I-\Delta)^{m} h=0 \text { weakly on } \mathbb{R}^{n} \backslash K \\
h=1 \text { on } K
\end{gathered}
$$

satisfies

$$
\langle H, h\rangle_{H^{m}\left(\mathbb{R}^{n}\right)}=\operatorname{Vol}(K)+\sum_{m / 2<j \leq m}(-1)^{j}\left(\begin{array}{c}
m \\
j
\end{array}\right) \lim _{r \downarrow 0} \int_{\partial K_{r}} \frac{\partial}{\partial \nu} \Delta^{j-1} h \mathrm{~d} S .
$$

In particular, taking $H=h$,

$$
\|h\|_{H^{m}\left(\mathbb{R}^{n}\right)}^{2}=\operatorname{Vol}(K)+\sum_{m / 2<j \leq m}(-1)^{j}\left(\begin{array}{c}
m \\
j
\end{array}\right) \lim _{r \downarrow 0} \int_{\partial K_{r}} \frac{\partial}{\partial \nu} \Delta^{j-1} h \mathrm{~d} S .
$$

If the boundary of $K$ is sufficiently regular to allow the invocation of boundary regularity for elliptic equations we may realise this formula more succinctly as

$$
\|h\|_{H^{m}\left(\mathbb{R}^{n}\right)}^{2}=\operatorname{Vol}(K)+\sum_{m / 2<j \leq m}(-1)^{j}\left(\begin{array}{c}
m \\
j
\end{array}\right) \int_{\partial K} \frac{\partial}{\partial \nu} \Delta^{j-1} h \mathrm{~d} S .
$$

The main virtue of Theorem 5 is that it explicitly demonstrates the dependence of the $m$-extremal energy upon the volume of $K$ and integrals over its boundary. It is analogous to the representation of the classical capacity $\inf \left\{\int|\nabla u|^{2}: u=1\right.$ on $\left.K\right\}$ as $\int_{\partial K} \frac{\partial u}{\partial \nu} \mathrm{d} S$ for $u$ satisfying $\Delta u=0$ off $K, u=1$ on $\partial K$ and $u \rightarrow 0$ at $\infty$. The 
fact that $\|h\|_{H^{m}\left(\mathbb{R}^{n}\right)}^{2}=\langle h, H\rangle_{H^{m}\left(\mathbb{R}^{n}\right)}$ for any $H \in H^{m}\left(\mathbb{R}^{n}\right)$ which is identically 1 on $K$ when $m=(n+1) / 2$ is more general and due to Meckes [17, Proposition 4.2. One may wonder whether the alternating sum in Theorem 5 reflects an underlying "Euler characteristic", and whether the individual terms appearing might have some separate meaning. It is also of interest to determine whether or not the solutions of the boundary value problems are always positive. See for example [6] for a discussion of such matters.

As mentioned in the Introduction, Leinster and Meckes have an alternative approach to calculating magnitudes. Indeed, Theorem 4.16 of [13], specialised to the case of Euclidean spaces, shows that up to a constant, the magnitude of a compact subset of $\mathbb{R}^{n}$ is the integral of its potential function, provided the potential function is in $L^{1}\left(\mathbb{R}^{n}\right)$. In the context of Theorem 5 and formula (15) this translates simply as

$$
\|h\|_{H^{m}\left(\mathbb{R}^{n}\right)}^{2}=\int_{\mathbb{R}^{n}} h .
$$

While this formula arguably offers some comptational advantages over formula (15), it does not bring out the important role that the boundary of $K$ plays. In this regard see also the last remark in Section 10.

\section{Combinatorial PREliminaries}

For our discussion of solutions to certain ordinary differential equations we shall need to consider some special polynomials which have a combinatorial flavour.

We define $g_{0}(t):=1$ and, given $g_{j}$, we define $g_{j+1}$ by

$$
g_{j+1}(t):=t^{3} g_{j}^{\prime}(t)+t g_{j}(t)
$$

Lemma 7. For $j \geq 1$ we have

$$
g_{j}(t)=\sum_{k} c_{k}^{j} t^{k}
$$

where $c_{k}^{j}=0$ for $k<j$ and $k \geq 2 j, c_{j}^{j}=1$ for all $j$, and each $c_{k}^{j}$ for $j \leq k \leq 2 j-1$ is a nonnegative integer.

The easy proof is left to the reader. The defining formula for $g_{j}$ leads us immediately to a recurrence relation for the coefficients $c_{k}^{j}$ :

Lemma 8. For $j+1 \leq k \leq 2 j-1$ we have

$$
c_{k+1}^{j+1}=(k-1) c_{k-1}^{j}+c_{k}^{j}
$$

and

$$
c_{2 j+1}^{j+1}=(2 j-1) c_{2 j-1}^{j} .
$$

Moreover for $j+1 \leq k \leq 2 j-1$ we have

$$
2 j c_{k+1}^{j+1}=(k-1) k c_{k-1}^{j}+2 k c_{k}^{j} .
$$


Proof. We have by definition

$$
\begin{aligned}
g_{j+1}(t) & =t^{3}\left(\sum_{k=j}^{2 j-1} c_{k}^{j} k t^{k-1}\right)+t\left(\sum_{k=j}^{2 j-1} c_{k}^{j} t^{k}\right) \\
& =\sum_{k=j}^{2 j-1} c_{k}^{j} k t^{k+2}+\sum_{k=j}^{2 j-1} c_{k}^{j} t^{k+1}=\sum_{k=j+1}^{2 j} c_{k-1}^{j}(k-1) t^{k+1}+\sum_{k=j}^{2 j-1} c_{k}^{j} t^{k+1} \\
& =c_{j}^{j} t^{j+1}+\sum_{k=j+1}^{2 j-1}\left(c_{k-1}^{j}(k-1)+c_{k}^{j}\right) t^{k+1}+c_{2 j-1}^{j}(2 j-1) t^{2 j+1} .
\end{aligned}
$$

On the other hand,

$$
g_{j+1}(t)=\sum_{k=j+1}^{2 j+1} c_{k}^{j+1} t^{k}=\sum_{k=j}^{2 j} c_{k+1}^{j+1} t^{k+1},
$$

and then (16) and (17) follow by comparing the two expressions. The final identity follows readily from the expression (18) below.

Pictorially, identity (16) can be represented as a Pascal triangle as follows, (with the first row representing $c_{1}^{1}$, the second $c_{2}^{2}, c_{3}^{2}$, etc.):

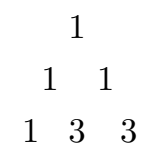

$\begin{array}{llll}1 & 6 & 15 & 15\end{array}$

$1 \quad 10 \quad 45105 \quad 105$

115105420945945

An explicit formula for $c_{k}^{j}$ for $j<k \leq 2 j-1$ is given by

$$
c_{k}^{j}=\frac{(k-1)(k-2) \cdots(2 j-k)}{2^{k-j}(k-j) !}
$$

and this follows immediately from equations (16) and (17) together with the fact that $c_{j}^{j}=1$ for all $j$.

The numbers $c_{k}^{j}$ are reminiscent of Stirling numbers and are sometimes called Bessel numbers of the first kind; the $g_{j}$ are related to Grosswald's polynomials. See [8] and 21. The $g_{j}$ can also be expressed in terms of generalised Laguerre polynomials and hypergeometric functions; we thank Jim Wright, Chris Smyth and Adri Olde Daalhuis for pointing these connections out to us. 


\section{Explicit RAdial SOlutions to $(I-\Delta)^{m} h=0$}

When $K$ is a Euclidean ball, the unique solution to problem (111) with $g=1$ is necessarily radial (since an average over rotates of a solution is also a solution). So in this section we seek radial solutions $h \in H^{m}\left(\mathbb{R}^{n}\right)$ to

$$
(I-\Delta)^{m} h=0 \text { on }\{|x|>R\}
$$

where $R>0$. Then we shall impose the extra (boundary) condition $h=1$ on $\{|x| \leq$ $R\}$. We are particularly interested in the case where $n$ is odd and $m=(n+1) / 2$. We shall systematically abuse notation and not distinguish between the function $h(|\cdot|)$ defined on $\{|x|>R\}$ in $\mathbb{R}^{n}$ and the function $h(\cdot)$ defined on $(R, \infty)$.

Recall that the action of the Laplacian in $\mathbb{R}^{n}$ on radial functions is given by

$$
\Delta f(r)=f^{\prime \prime}(r)+\frac{n-1}{r} f^{\prime}(r)
$$

and so we define, for an integer $\nu \geq 0$,

$$
\Delta_{\nu} f:=f^{\prime \prime}+\frac{2 \nu}{r} f^{\prime}
$$

and consider solutions of

$$
\left(I-\Delta_{\nu}\right)^{m} h=0
$$

on $(R, \infty)$ where $R>0$, with $h(|\cdot|) \in L^{2}\left(\mathbb{R}^{n}\right)$. (We hope that the the use of $\nu$ both as a proxy for $(n-1) / 2$ in this section and as a normal direction to $K$ in previous sections will not cause confusion.) If $n$ is odd and $\nu=(n-1) / 2$ this amounts to finding radial solutions in $L^{2}$ of

$$
(I-\Delta)^{m} h=0 \text { on }|x|>R .
$$

Let us set up some notation. We define, for $k \geq 0$,

$$
f_{k}(r):=\frac{e^{-r}}{r^{k}}
$$

We define $\psi_{0}(r)=e^{-r}$ and, for $j \geq 1$

$$
\psi_{j}(r):=\sum_{k=j}^{2 j-1} c_{k}^{j} f_{k}(r)=e^{-r} g_{j}(1 / r)
$$

where the $g_{j}$ are defined in Section 6

From Pascal's triangle for the coefficients $c_{k}^{j}$, (Lemma 8), we can write down the first few $\psi_{j}$ explicitly:

$$
\begin{aligned}
\psi_{0}=f_{0} & =e^{-r}, \\
\psi_{1}=f_{1} & =e^{-r} / r \\
\psi_{2}=f_{2}+f_{3} & =e^{-r}\left(r^{-2}+r^{-3}\right), \\
\psi_{3}=f_{3}+3 f_{4}+3 f_{5} & =e^{-r}\left(r^{-3}+3 r^{-4}+3 r^{-5}\right), \\
\psi_{4}=f_{4}+6 f_{5}+15 f_{6}+15 f_{7} & =e^{-r}\left(r^{-4}+6 r^{-5}+15 r^{-6}+15 r^{-7}\right), \\
\psi_{5}=f_{5}+10 f_{6}+45 f_{7}+105 f_{8}+105 f_{9} & =e^{-r}\left(r^{-5}+10 r^{-6}+45 r^{-7}+105 r^{-8}+105 r^{-9}\right)
\end{aligned}
$$


Lemma 9. For $k \geq 0$ and $\nu \geq 0$ we have

$$
\left(\Delta_{\nu}-I\right) f_{k}=2(k-\nu) f_{k+1}+k(k+1-2 \nu) f_{k+2} .
$$

In particular,

$$
\left(\Delta_{\nu}-I\right) f_{0}=-2 \nu f_{1}
$$

Proof. Let us calculate

$$
f_{k}^{\prime}=-\frac{e^{-r}}{r^{k}}-k \frac{e^{-r}}{r^{k+1}}=-f_{k}-k f_{k+1}
$$

so that

$$
\begin{aligned}
f_{k}^{\prime \prime} & =-f_{k}^{\prime}-k f_{k+1}^{\prime} \\
& =-\left(-f_{k}-k f_{k+1}\right)-k\left(-f_{k+1}-(k+1) f_{k+2}\right) \\
& =f_{k}+2 k f_{k+1}+k(k+1) f_{k+2} .
\end{aligned}
$$

Therefore

$$
\begin{aligned}
\left(\Delta_{\nu}-I\right) f_{k} & =f_{k}+2 k f_{k+1}+k(k+1) f_{k+2}-2 \nu\left(\frac{f_{k}}{r}+\frac{k f_{k+1}}{r}\right)-f_{k} \\
& =2 k f_{k+1}+k(k+1) f_{k+2}-2 \nu\left(f_{k+1}+k f_{k+2}\right) \\
& =2(k-\nu) f_{k+1}+k(k+1-2 \nu) f_{k+2} .
\end{aligned}
$$

Proposition 5. For $j \geq 0$ and $\nu \geq 0$ we have

$$
\left(\Delta_{\nu}-I\right) \psi_{j}=2(j-\nu) \psi_{j+1} .
$$

In particular,

$$
\left(\Delta_{\nu}-I\right) \psi_{\nu}=0 .
$$

Proof. The case $j=0$ is a special case of Lemma 9 For $j \geq 1$ we have

$$
\begin{aligned}
\left(\Delta_{\nu}-I\right) \psi_{j} & =\left(\Delta_{\nu}-I\right) \sum_{k=j}^{2 j-1} c_{k}^{j} f_{k}=\sum_{k=j}^{2 j-1} c_{k}^{j}\left(\Delta_{\nu}-I\right) f_{k} \\
& =\sum_{k=j}^{2 j-1} c_{k}^{j}\left[-2(\nu-k) f_{k+1}+k(k+1-2 \nu) f_{k+2}\right]
\end{aligned}
$$

by Lemma 9, Rearranging, this equals

$$
\begin{aligned}
\sum_{k=j}^{2 j-1} 2(k-\nu) c_{k}^{j} f_{k+1}+\sum_{k=j+1}^{2 j}(k-1)(k-2 \nu) c_{k-1}^{j} f_{k+1} \\
=2(j-\nu) c_{j}^{j} f_{j+1}+(2 j-1)(2 j-2 \nu) c_{2 j-1}^{j} f_{2 j+1}+ \\
\quad+\sum_{k=j+1}^{2 j-1}\left\{2(k-\nu) c_{k}^{j}+(k-1)(k-2 \nu) c_{k-1}^{j}\right\} f_{k+1} \\
=2(j-\nu)\left\{f_{j+1}+(2 j-1) c_{2 j-1}^{j} f_{2 j+1}\right\}+ \\
\quad+\sum_{k=j+1}^{2 j-1}\left\{2(k-\nu) c_{k}^{j}+(k-1)(k-2 \nu) c_{k-1}^{j}\right\} f_{k+1} .
\end{aligned}
$$


Now since

$$
2(j-\nu) \psi_{j+1}=2(j-\nu) \sum_{k=j+1}^{2 j+1} c_{k}^{j+1} f_{k}(r)=2(j-\nu) \sum_{k=j}^{2 j} c_{k+1}^{j+1} f_{k+1}(r)
$$

we see that the coefficients of $f_{j+1}$ agree (as $c_{j}^{j}=1$ for all $j$ ), and it is therefore enough to see that

$$
c_{2 j+1}^{j+1}=(2 j-1) c_{2 j-1}^{j}
$$

and, for $j+1 \leq k \leq 2 j-1$ and arbitrary $\nu$

$$
2(j-\nu) c_{k+1}^{j+1}=2(k-\nu) c_{k}^{j}+(k-1)(k-2 \nu) c_{k-1}^{j} .
$$

This latter splits as

$$
c_{k+1}^{j+1}=c_{k}^{j}+(k-1) c_{k-1}^{j}
$$

(coefficient of $\nu$ ) and

$$
2 j c_{k+1}^{j+1}=2 k c_{k}^{j}+(k-1) k c_{k-1}^{j}
$$

$(\nu=0)$. All of these are true identities by Lemma 8 .

Corollary 1. For $j \geq 0$ we have

$$
\psi_{j}^{\prime}(r)=-r \psi_{j+1}(r) .
$$

For $m \geq 1, \nu \geq 0$, and either $0 \leq j \leq \nu-m$ or $j>\nu$ we have

$$
\left(\Delta_{\nu}-I\right)^{m} \psi_{j}=2^{m}(j+m-1-\nu)(j+m-2-\nu) \cdots(j-\nu) \psi_{j+m},
$$

while for $\nu-m<j \leq \nu$ we have

$$
\left(\Delta_{\nu}-I\right)^{m} \psi_{j}=0 .
$$

Finally, for $m \geq 1, \nu \geq 0$ and $\psi=\sum_{j=0}^{\nu} \alpha_{j} \psi_{j}$,

$$
\Delta_{\nu}^{m} \psi=\sum_{j=0}^{\nu-m} \alpha_{j} 2^{m}(j+m-1-\nu) \cdots(j-\nu) \psi_{j+m}-\sum_{k=0}^{m-1}(-1)^{m-k}\left(\begin{array}{c}
m \\
k
\end{array}\right) \Delta_{\nu}^{k} \psi
$$

Proof. Write the first identity of Proposition [5 as

$$
\psi_{j}^{\prime \prime}+\frac{2 \nu}{r} \psi_{j}^{\prime}-\psi_{j}=2(j-\nu) \psi_{j+1}
$$

divide by $\nu$, and let $\nu \rightarrow \infty$ to obtain (20).

Let $j \geq 0$ and $m \geq 1$. By repeated application of Proposition 5 we obtain (21) and (22).

By the binomial theorem,

$$
\left(\Delta_{\nu}-I\right)^{m} \psi_{j}=\sum_{k=0}^{m}(-1)^{m-k}\left(\begin{array}{c}
m \\
k
\end{array}\right) \Delta_{\nu}^{k} \psi_{j}
$$

so that for $j \leq \nu-m$, by (21),

$$
\Delta_{\nu}^{m} \psi_{j}=2^{m}(j+m-1-\nu) \cdots(j-\nu) \psi_{j+m}-\sum_{k=0}^{m-1}(-1)^{m-k}\left(\begin{array}{c}
m \\
k
\end{array}\right) \Delta_{\nu}^{k} \psi_{j}
$$


while for $\nu-m<j \leq \nu$, by (22),

$$
\Delta_{\nu}^{m} \psi_{j}=-\sum_{k=0}^{m-1}(-1)^{m-k}\left(\begin{array}{c}
m \\
k
\end{array}\right) \Delta_{\nu}^{k} \psi_{j} .
$$

Finally (23) follows from (24) and (25) by taking linear combinations.

We shall use Corollary 1 to systematically turn the calculus into algebra when fitting the appropriate boundary conditions to the problem, and when calculating the right hand side of formula (15) in Theorem 5 in the radial case. Note that (23) tells us that for all $m \geq 1$, if $\psi=\sum_{j=0}^{\nu} \alpha_{j} \psi_{j}$, then $\Delta_{\nu}^{m} \psi$ can be expressed in terms of $\left\{\psi_{0}, \ldots, \psi_{\nu}\right\}$.

Returning now to the ordinary differential equation (19), we see that (22) from Corollary 1 tells us that the general solution to

$$
\left(I-\Delta_{\nu}\right)^{m} h=0
$$

on $\{r>0\}$ with $h(|\cdot|) \in L^{2}\left(\mathbb{R}^{n}\right)$ at infinity (for suitable $n$ ) is given by a linear combination of $\left\{\psi_{\nu}, \psi_{\nu-1}, \ldots, \psi_{\nu-m+1}\right\}$. (Routine calculations similar to those carried out at the beginning of this section reveal that the other $m$ linearly independent solutions will feature $e^{+r}$, and so will not have the requisite decay at infinity to belong to any $L^{2}\left(\mathbb{R}^{n}\right)$.)

In particular, taking $\nu=(n-1) / 2$ for $n$ odd, the general radial solution to

$$
(I-\Delta)^{m} h=0 \text { on } \mathbb{R}^{n} \backslash\{0\}
$$

with $h \in L^{2}\left(\mathbb{R}^{n}\right)$ at infinity is

$$
\alpha_{\nu} \psi_{v}(|x|)+\cdots+\alpha_{\nu-m+1} \psi_{\nu-m+1}(|x|)
$$

where the $\alpha$ 's are arbitrary scalars.

Specialising further, taking $m=(n+1) / 2$, we obtain:

Proposition 6. Suppose $n$ is odd. Then the general radial solution to

$$
(I-\Delta)^{(n+1) / 2} h=0 \text { on } \mathbb{R}^{n} \backslash\{0\}
$$

with $h \in L^{2}\left(\mathbb{R}^{n}\right)$ at infinity is

$$
h(x)=\alpha_{0} \psi_{0}(|x|)+\cdots+\alpha_{(n-1) / 2} \psi_{(n-1) / 2}(|x|)
$$

where the $\alpha$ 's are arbitrary scalars.

\section{The Algorithm For EXtremal Energy And Magnitude}

8.1. Fitting the boundary conditions. The next task is to determine the $\alpha_{j}$ 's which permit the solution $h=\sum_{j=0}^{(n-1) / 2} \alpha_{j} \psi_{j}(|\cdot|)$ of

$$
\begin{aligned}
(I-\Delta)^{(n+1) / 2} h & =0 \text { on }\{|x|>R\} \\
h & =1 \text { on }\{|x| \leq R\}
\end{aligned}
$$

given by Proposition 6 to lie in $H^{(n+1) / 2}\left(\mathbb{R}^{n}\right)$. This amounts to forcing it to satisfy appropriate boundary conditions at $|x|=R$. Now our radial solution $h$ belongs to $H^{(n+1) / 2}\left(\mathbb{R}^{n}\right)$ and so, by Lemma 4 its derivatives of order $j$ lie in $H^{n / 2-j}$ of spheres (for $0 \leq j \leq(n-1) / 2$ ) and moreover vary continuously with small changes in the radius of the sphere. Alternatively, the function $h$ defined on $(0, \infty)$ belongs 
in $H^{(n+1) / 2}(\mathbb{R})$ near $|x|=R$, and so by Sobolev embedding in the one-dimensional case, the derivatives of $h$ of order up to and including $(n-1) / 2$ are continuous at $R$. In any case, since $h=1$ on $\{|x| \leq R\}$, this manifestly forces the $(n+1) / 2$ conditions

$$
\begin{aligned}
h(R) & =1 \\
h^{\prime}(R) & =0 \\
\Delta h(R) & =0 \\
(\Delta h)^{\prime}(R) & =0 \\
\vdots & \\
\left(\Delta^{(n-1) / 4} h\right)(R) & =0 \text { or }\left(\Delta^{(n-3) / 4} h\right)^{\prime}(R)=0
\end{aligned}
$$

depending on whether $n-1$ or $n-3$ is a multiple of 4 . In either case, this gives us $(n+1) / 2$ linear conditions on the $(n+1) / 2$ unknowns $\alpha_{0}, \ldots, \alpha_{(n-1) / 2}$ which therefore determine them. (Since we know that there is a unique solution to the boundary value problem we know that the linear system (26) has a unique solution. Hence the system is guaranteed to be nonsingular - otherwise there would be either no solution or multiple solutions.) In practice, we shall need to use Corollary 1 in conjunction with the explicit formulae for the $\psi_{j}$ in order to evaluate the $\alpha_{j}$. As above, we write $\nu=(n-1) / 2$.

We shall use (23) from Corollary 1, directly on the equations involving $\Delta^{j} h(R)$, and (23) together with (20) on the equations involving $\left(\Delta^{j} h\right)^{\prime}(R)$.

The first equation is simply

$$
\alpha_{0} \psi_{0}(R)+\cdots+\alpha_{\nu} \psi_{\nu}(R)=1
$$

while subsequently we have, by (23) and the boundary conditions on the terms $h, \Delta h, \ldots(\Delta)^{m-1} h$,

$$
\Delta^{m} h(R)=2^{m} \sum_{j=0}^{\nu-m}(j+m-1-\nu)(j+m-2-\nu) \cdots(j-\nu) \alpha_{j} \psi_{j+m}(R)-(-1)^{m} .
$$

Since the terms $(j+m-r-\nu)$ are all negative and there are $m$ of them, the boundary condition $\Delta^{m} h(R)=0$ reduces this to

$$
2^{m} \sum_{j=0}^{\nu-m}(\nu-j)(\nu-j-1) \cdots(\nu-j-m+1) \alpha_{j} \psi_{j+m}(R)=1 .
$$

Similarly, the boundary condition $\left(\Delta^{m} h\right)^{\prime}(R)=0$ reduces to

$$
2^{m} \sum_{j=0}^{\nu-m}(\nu-j)(\nu-j-1) \cdots(\nu-j-m+1) \alpha_{j} \psi_{j+m+1}(R)=0 .
$$

Thus we have the $\nu+1=(n+1) / 2$ equations 


$$
\begin{aligned}
& \alpha_{0} \psi_{0}(R)+\alpha_{1} \psi_{1}(R) \\
& +\cdots+\alpha_{\nu} \psi_{\nu}(R) \quad=1 \\
& \alpha_{0} \psi_{1}(R)+\alpha_{1} \psi_{2}(R) \\
& +\cdots+\alpha_{\nu} \psi_{\nu+1}(R)=0 \\
& 2 \nu \alpha_{0} \psi_{1}(R)+2(\nu-1) \alpha_{1} \psi_{2}(R) \\
& +\cdots+2 \alpha_{\nu-1} \psi_{\nu}(R) \\
& =1 \\
& (27) \\
& 2 \nu \alpha_{0} \psi_{2}(R)+2(\nu-1) \alpha_{1} \psi_{3}(R) \\
& +\cdots+2 \alpha_{\nu-1} \psi_{\nu+1}(R)=0 \\
& 4 \nu(\nu-1) \alpha_{0} \psi_{2}(R)+4(\nu-1)(\nu-2) \alpha_{1} \psi_{3}(R)+\cdots+4.2 .1 \alpha_{\nu-2} \psi_{\nu}(R)=1 \\
& 4 \nu(\nu-1) \alpha_{0} \psi_{3}(R)+4(\nu-1)(\nu-2) \alpha_{1} \psi_{4}(R) \quad+\cdots+4.2 .1 \alpha_{\nu-2} \psi_{\nu+1}(R)=0 \\
& \vdots \quad=\quad \vdots
\end{aligned}
$$

to solve for the $\nu+1=(n+1) / 2$ unknowns $\alpha_{0}, \alpha_{1}, \ldots, \alpha_{\nu}$. (We know the system is nonsingular, but it is perhaps not so obvious that this is the case by direct inspection.) Notice that the "input" here involves only the values of $\psi_{0}(R), \ldots, \psi_{\nu+1}(R)$.

We implement the explicit solution of these equations in the first few cases corresponding to $n=1,3,5$ and 7 below.

8.2. Calculating the extremal energy. Once we have determined the $\alpha_{j}=$ $\alpha_{j}(R)$ which satisfy (27), we can use Theorem 5 to calculate the extremal energy $\|h\|_{H^{(n+1) / 2}\left(\mathbb{R}^{n}\right)}^{2}$ for $h=\alpha_{0} \psi_{0}+\cdots+\alpha_{\nu} \psi_{\nu}$. Indeed, we have, with $m=(n+1) / 2$,

$$
\begin{aligned}
\|h\|_{H^{(n+1) / 2}\left(\mathbb{R}^{n}\right)}^{2} & =\operatorname{Vol}(K)+\sum_{(n+1) / 4<j \leq(n+1) / 2}(-1)^{j}\left(\begin{array}{c}
\frac{n+1}{2} \\
j
\end{array}\right) \lim _{r \downarrow 0} \int_{\partial K_{r}} \frac{\partial}{\partial \nu} \Delta^{j-1} h \mathrm{~d} S \\
& =\omega_{n} R^{n}+\sigma_{n-1} R^{n-1} \sum_{(n+1) / 4<j \leq(n+1) / 2}(-1)^{j}\left(\begin{array}{c}
\frac{n+1}{2} \\
j
\end{array}\right)\left(\Delta^{j-1} h\right)^{\prime}(R) \\
& =\omega_{n} R^{n}\left(1+n R^{-1} \sum_{(n+1) / 4<j \leq(n+1) / 2}(-1)^{j}\left(\begin{array}{c}
\frac{n+1}{2} \\
j
\end{array}\right)\left(\Delta^{j-1} h\right)^{\prime}(R)\right)
\end{aligned}
$$

where $\omega_{n}$ is the volume of the unit ball in $\mathbb{R}^{n}$ and $\sigma_{n-1}=n \omega_{n}$ is the surface area of the unit sphere $\mathbb{S}^{n-1}$ in $\mathbb{R}^{n}$. We emphasise that by $\left(\Delta^{j-1} h\right)^{\prime}(R)$ we mean $\lim _{r \downarrow R}\left(\Delta^{j-1} h\right)^{\prime}(r)$ here and subsequently below.

The calculation of $\left(\Delta^{j-1} h\right)^{\prime}(R)$ is carried out using (23) from Corollary 1 Indeed, this gives us, for $j \geq 2$,

$$
\begin{aligned}
\Delta^{j-1} h= & 2^{j-1} \sum_{i=0}^{\nu-j+1}(i+j-2-\nu)(i+j-3-\nu) \cdots(i-\nu) \alpha_{i} \psi_{i+j-1} \\
& -\sum_{k=0}^{j-2}(-1)^{j-1-k}\left(\begin{array}{c}
j-1 \\
k
\end{array}\right) \Delta^{k} h .
\end{aligned}
$$

Now invoking $\psi_{j}^{\prime}(r)=-r \psi_{j+1}(r)$ (that is, (20) from Corollary 1), we obtain

$$
\begin{aligned}
\left(\Delta^{j-1} h\right)^{\prime}(R)= & -R 2^{j-1} \sum_{i=0}^{\nu-j+1}(i+j-2-\nu)(i+j-3-\nu) \cdots(i-\nu) \alpha_{i} \psi_{i+j}(R) \\
& -\sum_{k=0}^{j-2}(-1)^{j-1-k}\left(\begin{array}{c}
j-1 \\
k
\end{array}\right)\left(\Delta^{k} h\right)^{\prime}(R) .
\end{aligned}
$$


The boundary conditions imply that we have $\left(\Delta^{k} h\right)^{\prime}(R)=0$ for $0 \leq k \leq(\nu-2) / 2$ when $\nu$ is even, and $0 \leq k \leq(\nu-1) / 2$ when $\nu$ is odd. So in the $k$-sum we only need consider terms $k$ with $k>(\nu-1) / 2$, and therefore, recalling that $\nu=(n-1) / 2$, we have, for $j \geq 2$,

$$
\begin{aligned}
\left(\Delta^{j-1} h\right)^{\prime}(R)= & -R 2^{j-1} \sum_{i=0}^{\nu-j+1}(i+j-2-\nu) \cdots(i-\nu) \alpha_{i} \psi_{i+j}(R) \\
& -\sum_{(\nu-1) / 2<k \leq j-2}(-1)^{j-1-k}\left(\begin{array}{c}
j-1 \\
k
\end{array}\right)\left(\Delta^{k} h\right)^{\prime}(R) .
\end{aligned}
$$

This allows us to effectively compute the terms $\left(\Delta^{j-1} h\right)^{\prime}(R)$ recursively for $j \geq 2$ in terms of the values of $\psi_{0}(R), \ldots, \psi_{\nu+1}(R)$ and $\alpha_{0}, \ldots, \alpha_{\nu}$. We need this for those $j$ such that $(n+1) / 4<j \leq(n+1) / 2$. Once done, we can substitute into formula (28) for the extremal energy, and we carry this out explicitly in the cases $n=1,3$, 5 and 7 below.

8.3. Summary of the algorithm. We first solve the equations (27) for the $(n+$ 1) $/ 2$ unknowns $\alpha_{0}, \ldots, \alpha_{(n-1) / 2}$. Then we use formula (29) to calculate $\left(\Delta^{j-1} h\right)^{\prime}(R)$ for $(n+1) / 4<j \leq(n+1) / 2$, which we can substitute into formula (28), whose value we then compute to give the extremal energy. Finally we divide by $\omega_{n} n$ ! to obtain the magnitude.

8.4. Proof of Theorem 4, The discussion of the algorithm above immediately gives that the magnitude of the closed ball of radius $R$ in an odd-dimensional Euclidean space is a rational function of $R$. Indeed, modulo the multiplicative exponential term $e^{-R}$, all of the inputs $\psi_{0}(R), \ldots, \psi_{\nu}(R)$ are polynomials in $R^{-1}$ with nonnegative integer coefficients. The solutions $\alpha_{0}, \ldots, \alpha_{\nu}$ to our system of equations will be given, by Cramér's rule, by a ratio of two determinants, each of which is a polynomial in $\psi_{0}(R), \ldots, \psi_{\nu}(R)$ with integer coefficients, up to a multiplicative exponential term $e^{R}$. Inspection of formulae (29) and (28) indicates that the magnitude is given by $R^{n} / n$ ! plus an integer combination of various $\alpha_{r} \psi_{s}$ for $0 \leq r \leq(n-1) / 2$ and $0 \leq s \leq(n+1) / 2$. So the multiplicative exponential terms $e^{ \pm R}$ will cancel throughout in every instance, and we will be left with $R^{n} / n$ ! plus a rational function of $R$ with integer coefficients.

\section{ImPlementation of the ALgORithm}

Recall that we have

$$
\begin{aligned}
& \psi_{0}(r)=e^{-r} \\
& \psi_{1}(r)=\frac{e^{-r}}{r} \\
& \psi_{2}(r)=e^{-r}\left(\frac{1}{r^{2}}+\frac{1}{r^{3}}\right) \\
& \psi_{3}(r)=e^{-r}\left(\frac{1}{r^{3}}+\frac{3}{r^{4}}+\frac{3}{r^{5}}\right) \\
& \psi_{4}(r)=e^{-r}\left(\frac{1}{r^{4}}+\frac{6}{r^{5}}+\frac{15}{r^{6}}+\frac{15}{r^{7}}\right) .
\end{aligned}
$$


9.1. Case $n=1$. Notational fix: $\nu=0, m=1$.

Inputs: $\psi_{0}(R), \psi_{1}(R)$.

Outputs: $\alpha_{0}, h^{\prime}(R)$, extremal energy, magnitude.

The single equation for $\alpha_{0}$ is $\alpha_{0} \psi_{0}(R)=1$ which has solution $\alpha_{0}=1 / \psi_{0}(R)$.

Equation (28) requires $\left(\Delta^{j-1} h\right)^{\prime}(R)$ for $j=1$ only, which is simply $h^{\prime}(R)$, and by Corollary 1, equation (20), $h^{\prime}(R)=-R \alpha_{0} \psi_{1}(R)$.

Using (28), the extremal energy is given by

$$
\omega_{1} R\left(1+R^{-1}(-1)\left(\begin{array}{l}
1 \\
1
\end{array}\right) h^{\prime}(R)\right)=\omega_{1} R\left(1-R^{-1} h^{\prime}(R)\right)=\omega_{1} R\left(1+\alpha_{0} \psi_{1}(R)\right) .
$$

Substituting the value of $\alpha_{0}$ gives

$$
\omega_{1} R\left(1+\psi_{1}(R) / \psi_{0}(R)\right)=\omega_{1}(R+1) .
$$

Dividing by $1 ! \omega_{1}$ gives the magnitude of the closed interval $[-R, R]$ as

$$
R+1,
$$

in agreement with the results of [11] and [17].

9.2. Case $n=3$.

Notational fix: $\nu=1, m=2$.

Inputs: $\psi_{0}(R), \psi_{1}(R), \psi_{2}(R)$.

Outputs: $\alpha_{0}, \alpha_{1}, h^{\prime}(R),(\Delta h)^{\prime}(R)$, extremal energy, magnitude.

Our two equations are

$$
\begin{aligned}
& \alpha_{0} \psi_{0}(R)+\alpha_{1} \psi_{1}(R)=1 \\
& \alpha_{0} \psi_{1}(R)+\alpha_{1} \psi_{2}(R)=0
\end{aligned}
$$

which have solution

$$
\begin{aligned}
\alpha_{0} & =\frac{\psi_{2}(R)}{\psi_{0}(R) \psi_{2}(R)-\psi_{1}(R)^{2}} \\
\alpha_{1} & =\frac{-\psi_{1}(R)}{\psi_{0}(R) \psi_{2}(R)-\psi_{1}(R)^{2}} .
\end{aligned}
$$

Now $\psi_{0}(R) \psi_{2}(R)-\psi_{1}(R)^{2}=e^{-2 R}\left(R^{-2}+R^{-3}-R^{-2}\right)=e^{-2 R} R^{-3}$, so

$$
\begin{aligned}
\alpha_{0} & =e^{R}(R+1) \\
\alpha_{1} & =-e^{R} R^{2} .
\end{aligned}
$$

Note the appearance of the expression $R+1$ (as displayed in the previous subsection) in the formula for $\alpha_{0}$.

Equation (28) requires $\left(\Delta^{j-1} h\right)^{\prime}(R)$ for $j=2$ only, and by formula (29) we have

$$
(\Delta h)^{\prime}(R)=-R(2)^{1}(-1) \alpha_{0} \psi_{2}(R)-0=2 R \alpha_{0} \psi_{2}(R)
$$

Using (28), the extremal energy is given by

$$
\begin{gathered}
\omega_{3} R^{3}\left(1+3 R^{-1}(-1)^{2}\left(\begin{array}{l}
2 \\
2
\end{array}\right)(\Delta h)^{\prime}(R)\right)=\omega_{3} R^{3}\left(1+3 R^{-1}(\Delta h)^{\prime}(R)\right) \\
=\omega_{3} R^{3}\left(1+6 \alpha_{0} \psi_{2}(R)\right) .
\end{gathered}
$$


If we substitute in the explicit values for $\alpha_{0}$ and $\psi_{2}(R)$ we obtain

$$
=\omega_{3}\left(R^{3}+6 R^{2}+12 R+6\right),
$$

which upon dividing by $3 ! \omega_{3}$ gives the magnitude of the closed ball of radius $R$ in $\mathbb{R}^{3}$ as

$$
\frac{1}{3 !}\left(R^{3}+6 R^{2}+12 R+6\right)=\frac{R^{3}}{3 !}+R^{2}+2 R+1 .
$$

This verifies the Leinster-Willerton convex magnitude conjecture for balls in $\mathbb{R}^{3}$.

9.3. Case $n=5$.

Notational fix: $\nu=2, m=3$.

Inputs: $\psi_{0}(R), \ldots, \psi_{3}(R)$.

Outputs: $\alpha_{0}, \alpha_{1}, \alpha_{2}, h^{\prime}(R), \Delta h^{\prime}(R),\left(\Delta^{2} h\right)^{\prime}(R)$, extremal energy, magnitude.

Our three equations are

$$
\begin{array}{clll}
\alpha_{0} \psi_{0}(R)+\alpha_{1} \psi_{1}(R) & +\alpha_{2} \psi_{2}(R) & = & 1 \\
\alpha_{0} \psi_{1}(R)+\alpha_{1} \psi_{2}(R) & +\alpha_{2} \psi_{3}(R) & = & 0 \\
4 \alpha_{0} \psi_{1}(R)+2 \alpha_{1} \psi_{2}(R) & +0 & = & 1
\end{array}
$$

which upon substitution of the values of $\psi_{j}(R)$ become

$$
\begin{array}{clll}
\alpha_{0}+\alpha_{1} R^{-1} & +\alpha_{2}\left(R^{-2}+R^{-3}\right) & = & e^{R} \\
\alpha_{0} R^{-1}+\alpha_{1}\left(R^{-2}+R^{-3}\right) & +\alpha_{2}\left(R^{-3}+3 R^{-4}+3 R^{-5}\right) & = & 0 \\
\alpha_{0} 4 R^{-1}+\alpha_{1} 2\left(R^{-2}+R^{-3}\right) & +0 & = & e^{R} .
\end{array}
$$

A visit to matrixcalc.org confirms that the solution of these equations is given by

$$
\alpha_{j}=\frac{e^{R} \beta_{j}}{2(R+3)}
$$

where

$$
\begin{aligned}
& \beta_{0}=6+12 R+6 R^{2}+R^{3} \\
& \beta_{1}=-R^{2}\left(12+9 R+2 R^{2}\right) \\
& \beta_{2}=(2+R) R^{4} .
\end{aligned}
$$

Note that the system is singular when $R=-3$, and note the appearance of the expression $R^{3} / 6+R^{2}+2 R+1$ (as displayed in the previous subsection) in the formula for $\alpha_{0}$.

Equation (28) requires $\left(\Delta^{j-1} h\right)^{\prime}(R)$ for $j=2$ and 3 , and by formula (29) we have (case $j=2$ )

$$
(\Delta h)^{\prime}(R)=-R .2 \sum_{i=0}^{1}(i-2) \alpha_{i} \psi_{i+2}(R)+0=2 R\left(2 \alpha_{0} \psi_{2}(R)+\alpha_{1} \psi_{3}(R)\right),
$$


and, (case $j=3$ ),

$$
\begin{aligned}
\left(\Delta^{2} h\right)^{\prime}(R) & =-R 2^{2}(-1)(-2) \alpha_{0} \psi_{3}(R)-(-1)^{1}\left(\begin{array}{l}
2 \\
1
\end{array}\right)(\Delta h)^{\prime}(R) \\
& =-8 R \alpha_{0} \psi_{3}(R)+4 R\left(2 \alpha_{0} \psi_{2}(R)+\alpha_{1} \psi_{3}(R)\right) \\
& =-8 R \alpha_{0} \psi_{3}(R)+8 R \alpha_{0} \psi_{2}(R)+4 R \alpha_{1} \psi_{3}(R) .
\end{aligned}
$$

Using (28), the extremal energy is given by

$$
\begin{aligned}
\omega_{5} & R^{5}\left(1+5 R^{-1}\left\{\left(\begin{array}{l}
3 \\
2
\end{array}\right)(\Delta h)^{\prime}(R)-\left(\begin{array}{l}
3 \\
3
\end{array}\right)\left(\Delta^{2} h\right)^{\prime}(R)\right\}\right) \\
& =\omega_{5} R^{5}\left(1+5 R^{-1}\left\{3(\Delta h)^{\prime}(R)-\left(\Delta^{2} h\right)^{\prime}(R)\right\}\right) \\
& =\omega_{5} R^{5}\left(1+5\left\{3\left[4 \alpha_{0} \psi_{2}(R)+2 \alpha_{1} \psi_{3}(R)\right]-\left[-8 \alpha_{0} \psi_{3}(R)+8 \alpha_{0} \psi_{2}(R)+4 \alpha_{1} \psi_{3}(R)\right]\right\}\right) \\
& =\omega_{5} R^{5}\left(1+5\left\{4 \alpha_{0} \psi_{2}(R)+8 \alpha_{0} \psi_{3}(R)+2 \alpha_{1} \psi_{3}(R)\right\}\right) .
\end{aligned}
$$

Substituting in the explicit values for $\psi_{2}(R)$ and $\psi_{3}(R)$ we see that this expression equals

$$
\begin{aligned}
& \omega_{5}\left(R^{5}+5 e^{-R} R^{5}\left[\left(4 \alpha_{0}\left(R^{-2}+R^{-3}\right)+8 \alpha_{0}\left(R^{-3}+3 R^{-4}+3 R^{-5}\right)+2 \alpha_{1}\left(R^{-3}+3 R^{-4}+3 R^{-5}\right)\right]\right)\right. \\
= & \omega_{5}\left(R^{5}+5 e^{-R}\left[\alpha_{0}\left(4 R^{3}+12 R^{2}+24 R+24\right)+\alpha_{1}\left(2 R^{2}+6 R+6\right)\right]\right) .
\end{aligned}
$$

Now plugging in the explicit values for $\alpha_{0}$ and $\alpha_{1}$, we obtain

$\omega_{5} R^{5}+\frac{5 \omega_{5}}{2(R+3)}\left(\left(6+12 R+6 R^{2}+R^{3}\right)\left(4 R^{3}+12 R^{2}+24 R+24\right)-R^{2}\left(12+9 R+2 R^{2}\right)\left(2 R^{2}+6 R+6\right)\right)$

which, after some simplification and cancellation in the highest order terms, gives that the extremal energy is given by

$$
\omega_{5} R^{5}+5 \omega_{5} \frac{72+216 R+216 R^{2}+105 R^{3}+27 R^{4}+3 R^{5}}{R+3} .
$$

Dividing by $c_{5}=5 ! \omega_{5}$ we obtain that the magnitude of the ball of radius $R$ in $\mathbb{R}^{5}$ is

$$
\frac{R^{5}}{5 !}+\frac{3 R^{5}+27 R^{4}+105 R^{3}+216 R^{2}+216 R+72}{24(R+3)} .
$$

Note that when $R=0$ we obtain the value 1 , but when $R>0$ this quantity is strictly greater than the conjectured value of

$$
\frac{1}{5 !} R^{5}+\frac{1}{9} R^{4}+\frac{2}{3} R^{3}+2 R^{2}+\frac{8}{3} R+2 R^{2}+1 .
$$

We can expand our formula when $R>3$ as

$$
\frac{1}{5 !} R^{5}+\frac{1}{8} R^{4}+\frac{3}{4} R^{3}+\frac{17}{8} R^{2}+\frac{21}{8} R+\frac{9}{8}+O\left(\frac{1}{R}\right)
$$

as $R \rightarrow \infty$, but the coefficients (other than that of $R^{5}$ ) do not agree either with those conjectured by Leinster and Willerton. 
9.4. Case $n=7$.

Notational fix: $\nu=3, m=4$.

Inputs: $\psi_{0}(R), \ldots, \psi_{4}(R)$.

Outputs: $\alpha_{0}, \ldots, \alpha_{3}, h^{\prime}(R), \ldots,\left(\Delta^{3} h\right)^{\prime}(R)$, extremal energy, magnitude.

For brevity of expression we suppress the multiplicative factors $e^{ \pm R}$ which should appear below, and which ultimately cancel as described above. We have the four equations

$$
\left\{\begin{array}{l}
\alpha_{0} \psi_{0}(R)+\alpha_{1} \psi_{1}(R)+\alpha_{2} \psi_{2}(R)+\alpha_{3} \psi_{3}(R)=1 \\
\alpha_{0} \psi_{1}(R)+\alpha_{1} \psi_{2}(R)+\alpha_{2} \psi_{3}(R)+\alpha_{3} \psi_{4}(R)=0 \\
6 \alpha_{0} \psi_{1}(R)+4 \alpha_{1} \psi_{2}(R)+2 \alpha_{2} \psi_{3}(R)=1 \\
3 \alpha_{0} \psi_{2}(R)+2 \alpha_{1} \psi_{3}(R)+\alpha_{2} \psi_{4}(R)=0
\end{array}\right.
$$

with solution

$$
\begin{aligned}
& \alpha_{0}=\frac{360+1080 R+1080 R^{2}+525 R^{3}+135 R^{4}+18 R^{5}+R^{6}}{360+288 R+72 R^{2}+6 R^{3}}, \\
& \alpha_{1}=-\frac{360 R^{2}+555 R^{3}+345 R^{4}+105 R^{5}+16 R^{6}+R^{7}}{120+96 R+24 R^{2}+2 R^{3}}, \\
& \alpha_{2}=\frac{120 R^{4}+150 R^{5}+66 R^{6}+13 R^{7}+R^{8}}{120+96 R+24 R^{2}+2 R^{3}}, \\
& \alpha_{3}=-\frac{24 R^{6}+27 R^{7}+9 R^{8}+R^{9}}{360+288 R+72 R^{2}+6 R^{3}}
\end{aligned}
$$

as another visit to matrixcalc.org confirms.

Note that the numerator in the formula for $\alpha_{0}$ is

$$
360+1080 R+1080 R^{2}+525 R^{3}+135 R^{4}+18 R^{5}+R^{6}
$$

whereas the magnitude of the ball of radius $R$ in $\mathbb{R}^{5}$ (calculated in the previous subsection) is

$$
\begin{aligned}
& \frac{R^{5}}{5 !}+\frac{3 R^{5}+27 R^{4}+105 R^{3}+216 R^{2}+216 R+72}{24(R+3)} \\
= & \frac{360+1080 R+1080 R^{2}+525 R^{3}+135 R^{4}+18 R^{5}+R^{6}}{120(R+3)},
\end{aligned}
$$

with the same numerator as $\alpha_{0}$.

From equation (28) the extremal energy is given by

$$
\begin{aligned}
& \omega_{7} R^{7}+7 \omega_{7} R^{6}\left(-\left(\begin{array}{l}
4 \\
3
\end{array}\right)\left(\Delta^{2} u\right)^{\prime}(R)+\left(\begin{array}{l}
4 \\
4
\end{array}\right)\left(\Delta^{3} u\right)^{\prime}(R)\right) \\
= & \omega_{7}\left(R^{7}+7 R^{6}\left(-4\left(\Delta^{2} u\right)^{\prime}(R)+\left(\Delta^{3} u\right)^{\prime}(R)\right)\right. \\
= & \omega_{7}\left(R^{7}-28 R^{6}\left(\Delta^{2} u\right)^{\prime}(R)+7 R^{6}\left(\Delta^{3} u\right)^{\prime}(R)\right) \\
= & \omega_{7}\left(R^{7}+7 R^{6}\left\{\left(\Delta^{3} u\right)^{\prime}(R)-4\left(\Delta^{2} u\right)^{\prime}(R)\right\}\right) .
\end{aligned}
$$

So we need to calculate $\left(\Delta^{2} u\right)^{\prime}(R)$ and $\left(\Delta^{3} u\right)^{\prime}(R)$. 
Using the recurrence relation (29) with $j=3$ gives

$$
\begin{aligned}
\left(\Delta^{2} h\right)^{\prime}(R) & =-R 2^{2} \sum_{i=0}^{1}(i-2)(i-3) \alpha_{i} \psi_{i+3}(R)+0 \\
& =-4 R\left(6 \alpha_{0} \psi_{3}(R)+2 \alpha_{1} \psi_{4}(R)\right) \\
& =-8 R\left(3 \alpha_{0} \psi_{3}(R)+\alpha_{1} \psi_{4}(R)\right) .
\end{aligned}
$$

Next, using the recurrence relation (29) with $j=4$ gives

$$
\begin{aligned}
\left(\Delta^{3} h\right)^{\prime}(R) & =6 R 2^{3} \alpha_{0} \psi_{4}(R)+\left(\begin{array}{l}
3 \\
2
\end{array}\right)\left(\Delta^{2} h\right)^{\prime}(R) \\
& =48 R \alpha_{0} \psi_{4}(R)+3\left(-8 R\left(3 \alpha_{0} \psi_{3}(R)+\alpha_{1} \psi_{4}(R)\right)\right) \\
& =48 R \alpha_{0} \psi_{4}(R)-24 R\left(3 \alpha_{0} \psi_{3}(R)+\alpha_{1} \psi_{4}(R)\right) \\
& =24 R\left(2 \alpha_{0} \psi_{4}(R)-3 \alpha_{0} \psi_{3}(R)-\alpha_{1} \psi_{4}(R)\right) .
\end{aligned}
$$

Now

$$
\begin{aligned}
3 \alpha_{0} \psi_{3}+\alpha_{1} \psi_{4}= & \frac{360+1080 R+1080 R^{2}+525 R^{3}+135 R^{4}+18 R^{5}+R^{6}}{120+96 R+24 R^{2}+2 R^{3}} \times \frac{3+3 R+R^{2}}{R^{5}} \\
& -\frac{360+555 R+345 R^{2}+105 R^{3}+16 R^{4}+R^{5}}{120+96 R+24 R^{2}+2 R^{3}} \times \frac{15+15 R+6 R^{2}+R^{3}}{R^{5}} \\
= & -\frac{4320+9405 R+8820 R^{2}+4545 R^{3}+1380 R^{4}+246 R^{5}+24 R^{6}+R^{7}}{R^{5}\left(120+96 R+24 R^{2}+2 R^{3}\right)}
\end{aligned}
$$

(noticing the cancellation in the $R^{8}$ terms), and so

$$
\left(\Delta^{2} u\right)^{\prime}(R)=\frac{8\left(4320+9405 R+8820 R^{2}+4545 R^{3}+1380 R^{4}+246 R^{5}+24 R^{6}+R^{7}\right)}{R^{4}\left(120+96 R+24 R^{2}+2 R^{3}\right)} .
$$

Moreover

$3 \psi_{3}-2 \psi_{4}=\frac{3}{R^{3}}+\frac{9}{R^{4}}+\frac{9}{R^{5}}-\frac{2}{R^{4}}-\frac{12}{R^{5}}-\frac{30}{R^{6}}-\frac{30}{R^{7}}=\frac{-30-30 R-3 R^{2}+7 R^{3}+3 R^{4}}{R^{7}}$,

so that

equals

$$
\alpha_{0}\left(3 \psi_{3}-2 \psi_{4}\right)+\alpha_{1} \psi_{4}
$$

$$
\begin{aligned}
& \frac{360+1080 R+1080 R^{2}+525 R^{3}+135 R^{4}+18 R^{5}+R^{6}}{360+288 R+72 R^{2}+6 R^{3}} \times \frac{-30-30 R-3 R^{2}+7 R^{3}+3 R^{4}}{R^{7}} \\
& -\frac{1080 R^{2}+1665 R^{3}+1035 R^{4}+315 R^{5}+48 R^{6}+3 R^{7}}{360+288 R+72 R^{2}+6 R^{3}} \times \frac{15+15 R+6 R^{2}+R^{3}}{R^{7}} \\
= & -\frac{10800+43200 R+82080 R^{2}+90045 R^{3}+61380 R^{4}+26685 R^{5}+7380 R^{6}+1254 R^{7}+120 R^{8}+5 R^{9}}{R^{7}\left(360+288 R+72 R^{2}+6 R^{3}\right)}
\end{aligned}
$$

(noticing the cancellation in the $R^{10}$ terms), which gives that

$$
\left(\Delta^{3} u\right)^{\prime}(R)
$$


equals

$\frac{24\left(10800+43200 R+82080 R^{2}+90045 R^{3}+61380 R^{4}+26685 R^{5}+7380 R^{6}+1254 R^{7}+120 R^{8}+5 R^{9}\right)}{R^{6}\left(360+288 R+72 R^{2}+6 R^{3}\right)}$.

$$
R^{6}\left(360+288 R+72 R^{2}+6 R^{3}\right)
$$

Combining these, we see that

$$
R^{6}\left(\Delta^{3} u\right)^{\prime}(R)-4 R^{6}\left(\Delta^{2} u\right)^{\prime}(R)
$$

equals

$$
\begin{aligned}
& \frac{24\left(10800+43200 R+82080 R^{2}+90045 R^{3}+61380 R^{4}+26685 R^{5}+7380 R^{6}+1254 R^{7}+120 R^{8}+5 R^{9}\right)}{360+288 R+72 R^{2}+6 R^{3}} \\
& -\frac{96\left(4320 R^{2}+9405 R^{3}+8820 R^{4}+4545 R^{5}+1380 R^{6}+246 R^{7}+24 R^{8}+R^{9}\right)}{360+288 R+72 R^{2}+6 R^{3}} \\
= & \frac{43200+172800 R+259200 R^{2}+209700 R^{3}+104400 R^{4}+34020 R^{5}+7440 R^{6}+1080 R^{7}+96 R^{8}+4 R^{9}}{60+48 R+12 R^{2}+R^{3}} .
\end{aligned}
$$

Finally, we insert this into (32) and divide by $7 ! \omega_{7}$ to see that the magnitude of the closed ball of radius $R$ in $\mathbb{R}^{7}$ is given by

$$
\frac{R^{7}}{7 !}+\frac{\frac{1}{180} R^{9}+\frac{2}{15} R^{8}+\frac{3}{2} R^{7}+\frac{31}{3} R^{6}+\frac{189}{4} R^{5}+145 R^{4}+\frac{1165}{4} R^{3}+360 R^{2}+240 R+60}{R^{3}+12 R^{2}+48 R+60} .
$$

\section{Concluding Remarks}

1. The three-dimensional convex conjecture predicts that the magnitude of the convex body $K$ should be

$$
\frac{V_{0}(K)}{0 ! \omega_{0}}+\frac{V_{1}(K)}{1 ! \omega_{1}}+\frac{V_{2}(K)}{2 ! \omega_{2}}+\frac{V_{3}(K)}{3 ! \omega_{3}}
$$

where $V_{j}(K)$ is the $j$ 'th intrinsic volume and $\omega_{0}=1, \omega_{1}=2, \omega_{2}=\pi$ and $\omega_{3}=4 \pi / 3$. Now $V_{0}(K)=1, V_{2}(K)=\operatorname{Surf}(\partial K) / 2, V_{3}(K)=\operatorname{Vol}(K)$ and $V_{1}(K)=P_{2}(K) / \pi$ where $P_{2}(K)$ is the coefficient of $t^{2}$ in the degree 3 polynomial vol $\left(K+t B_{1}\right)$. So the magnitude of $K$ should be

$$
\frac{\operatorname{Vol}(K)}{8 \pi}+\frac{\operatorname{Surf}(\partial K)}{4 \pi}+\frac{V_{1}(K)}{2}+1 .
$$

In the notation of Theorem 5, the question of validity of the convex magnitude conjecture reduces to whether for the solution $h \in H^{2}\left(\mathbb{R}^{3}\right)$ of the boundary value problem $(I-\Delta)^{2} h=0$ off $K, h=1$ on $K$, we have

$$
\frac{1}{8 \pi} \int_{\partial K} \frac{\partial \Delta h}{\partial \nu} \mathrm{d} S=\frac{\operatorname{surf}(\partial K)}{4 \pi}+\frac{V_{1}(K)}{2}+1 .
$$

When $K$ is the cuboid $\left[-R_{1}, R_{1}\right] \times\left[-R_{2}, R_{2}\right] \times\left[-R_{3}, R_{3}\right]$ we have $V_{1}(K)=2\left(R_{1}+\right.$ $R_{2}+R_{3}$ ), so that we expect the magnitude of $K$ to be

$$
\frac{R_{1} R_{2} R_{3}}{\pi}+\frac{\left(R_{1} R_{2}+R_{2} R_{3}+R_{3} R_{1}\right)}{2 \pi}+\left(R_{1}+R_{2}+R_{3}\right)+1 .
$$


In particular in the case of the cuboid do we have

$$
\frac{1}{8 \pi} \int_{\partial K} \frac{\partial \Delta h}{\partial \nu} \mathrm{d} S=\frac{\left(R_{1} R_{2}+R_{2} R_{3}+R_{3} R_{1}\right)}{2 \pi}+\left(R_{1}+R_{2}+R_{3}\right)+1 ?
$$

Even when $R_{j}=R$ for $j=1,2,3$, why should the quantity

$$
\frac{1}{8 \pi} \int_{\partial K} \frac{\partial \Delta h}{\partial \nu} \mathrm{d} S
$$

be a polynomial of degree 2 , still less the specific polynomial $3 R^{2} / 2 \pi+3 R+1$ ? One of the difficulties in addressing this problem lies in not having explicit formulae for solutions of these types of boundary value problems in the absence of spherical symmetry.

On the other hand, matters may be better if we instead consider ellipsoids. Khavinson and Lundberg [9] note that ellipsoidal harmonics can be used to study elliptic boundary value problems in the interior of ellipsoids. It may be possible to use them in the current context to obtain explicit solutions of $(I-\Delta)^{2} u=0$ in the exterior of an ellipsoid in $\mathbb{R}^{3}$ which might then lead to explicit formulae for the magnitudes of ellipsoids in $\mathbb{R}^{3}$. Note however that the mixed volumes, even the surface area, of a general ellipsoid in $\mathbb{R}^{3}$ are not expressible as elementary functions of the semi-axes, but instead involve elliptic integrals.

2. The methods presented here can be extended to obtain explicit expressions for the magnitude of general compact sets possessing spherical symmetry.

3. An as-yet-unexplained (empirical) phenomenon is that in the formula for the coefficient $\alpha_{0}(R)$ in the $n$-dimensional setting, with $n$ odd and small, the formula for magnitude of the ball of radius $R$ in $\mathbb{R}^{n-2}$ makes a rather mysterious appearance, see the previous section. Further observations of this kind have been made by Willerton, (private communication), who has also developed a more streamlined approach to solving the ODE (19) and fitting the boundary conditions. This leads to a more symmetric system of linear equations for the unknowns $\alpha_{j}$. Using the new approach to calculating magnitude of Leinster and Meckes (13, Theorem 4.16), he is able to derive a formula for the magnitude of a ball in odd dimensions expressed in terms of the reverse Bessel polynomials. This formula can be re-cast in a reasonably succinct and natural form in odd dimensions at least up to and including 39 (where the coefficients of the numerator in the magnitude total over 60,000 digits).

4. The methods here can also be used to study the extremal energies or Bessel-like capacities

$$
C_{m}(K, \lambda):=\inf \left\{\left\|(\lambda I-\Delta)^{m / 2} h\right\|_{L^{2}\left(\mathbb{R}^{n}\right)}^{2}: h \equiv 1 \text { on } K\right\}
$$

for $\lambda>0$ for convex bodies $K$ when $m \in \mathbb{N}$ and to calculate them explicitly when $K$ is a ball. Indeed, a simple scaling argument shows that

$$
C_{m}(K, \lambda)=\lambda^{m-n / 2} C_{m}\left(\lambda^{1 / 2} K, 1\right)
$$

and we have shown the asymptotic behaviour of $C_{(n+1) / 2}(K, 1)$ and how to calculate it explicitly for balls above. In particular, $C_{(n+1) / 2}(K, \lambda) \sim \lambda^{1 / 2}$ as $\lambda \rightarrow 0$ and $C_{(n+1) / 2}(K, \lambda) \sim \lambda^{(n+1) / 2} \operatorname{Vol}(K) / n ! \omega_{n}$ as $\lambda \rightarrow \infty$. When $m=1$ and $n=3$ for example, we can calculate using Theorem 5 and the formula for $\psi_{1}$ that $C_{1}(B(0, R), 1)$ 
is simply $4 \pi\left(R^{3} / 3+R+1\right)$. So

$$
\begin{aligned}
C_{1}(B(0, R), \lambda) & =\lambda^{-1 / 2} C_{1}\left(B\left(0, \lambda^{1 / 2} R\right), 1\right) \\
& =4 \pi \lambda^{-1 / 2}\left(\lambda^{3 / 2} R^{3} / 3+\lambda^{1 / 2} R+1\right) \\
& =4 \pi \lambda R^{3} / 3+4 \pi R+4 \pi \lambda^{-1 / 2} .
\end{aligned}
$$

5. There remains the possibility that a reformulated version of the convex magnitude conjecture might be true if we replaced magnitude by some closely related notion which coincides with it in dimensions one and three, and is strictly smaller than it for nontrivial balls in odd dimensions five and above. It would be very interesting to find a such a notion. The notion of maximum diversity

$$
|X|_{+}:=\frac{1}{n ! \omega_{n}} \inf \left\{\|f\|_{H^{(n+1) / 2}\left(\mathbb{R}^{n}\right)}^{2}: f \in H^{(n+1) / 2}\left(\mathbb{R}^{n}\right), f \geq 1 \text { on } X\right\}
$$

found for example in 16, 17, and the references therein, and which is much more intune than magnitude with classical potential theory, unfortunately does not satisfy these criteria. One should note the parallel between the definition of maximum diversity and certain obstacle problems for higher-order operators (see for example [3] and [4) though this does not seem to have been exploited yet.) We thank E. Milakis for bringing this parallel to our attention.

6. In forthcoming work, H. Gimperlein and M. Goffeng [7] use methods from semiclassical analysis together with our Theorem 5 to exhibit an asymptotic expansion of the magnitude $|R X|$ as $R \rightarrow \infty$, where $X \subseteq \mathbb{R}^{n}$ is open and bounded with smooth boundary, when $n \geq 3$ is odd. In particular, they prove

$$
|R X|=\frac{\operatorname{Vol}(X)}{n ! \omega_{n}} R^{n}+\frac{(n+1) \operatorname{Surf}(\partial X)}{2 n ! \omega_{n}} R^{n-1}+c R^{n-2}+o\left(R^{n-2}\right),
$$

where $c$ is proportional to an integral of a curvature of $\partial X$. (The coefficient of $R^{n-1}$ differs from the prediction of Leinster and Willerton unless $n=3$.)

\section{REFERENCES}

[1] D. R. Adams and L. I. Hedberg, Function Spaces and Potential Theory, Volume 314 of Grundlehren der Mathematischen Wissenschaften, Springer-Verlag, Berlin, 1996.

[2] R. A. Adams and J. J. F. Fournier, Sobolev spaces, Second edition, Pure and Applied Mathematics (Amsterdam), 140. Elsevier/Academic Press, Amsterdam, 2003.

[3] L. A. Caffarelli and A. Friedman, The obstacle problem for the biharmonic operator, Ann. Scu. Norm. Sup. Pisa 6 (1979) $151-184$.

[4] L. A. Caffarelli, A. Friedman and A. Torelli, The two obstacle problem for the biharmonic operator, Pacific J. Math., 103 (1982) 325 - 335.

[5] L. C. Evans, Partial differential equations, Second edition, Graduate Studies in Mathematics, 19. American Mathematical Society, Providence, RI, 2010.

[6] F. Gazzola, H-C. Grunau and G. Sweers, Polyharmonic boundary value problems, Springer 2015.

[7] H. Gimperlein and M. Goffeng, Semiclassical expansion of magnitudes of domains in Euclidean spaces, manuscript in preparation

[8] E. Grosswald, Bessel Polynomials, Lecture Notes Math. vol. 698 1978, p. 18.

[9] D. Khavinson and E. Lundberg, A Tale of ellipsoids in potential theory, Notices of the Amer. Math. Soc. 61, no.2 (2014) 148-156

[10] D. A. Klain and G.-C. Rota, Introduction to Geometric Probability Lezioni Lincee, Cambridge University Press, Cambridge, 1997. 
[11] T. Leinster, The magnitude of metric spaces, Doc. Math. 18 (2013) 857-905.

[12] T. Leinster and S. Willerton, On the asymptotic magnitude of subsets of Euclidean space, Geom. Dedicata 164(1) (2013) 287-310.

[13] T. Leinster and M. Meckes, The magnitude of a metric space: from category theory to geometric measure theory, arXiv:1606.00095 [math.MG]

[14] P. McMullen, Valuations and Euler-type relations on certain classes of convex polytopes, Proc. Lond. Math. Soc. 35 (1977) 113-135.

[15] P. McMullen, Continuous translation invariant valuations on the space of compact convex sets, Archiv der Mathematik 34 (1980) 377-384.

[16] M. Meckes, Positive definite metric spaces, Positivity, 17(3) (2013) 733-757.

[17] M. Meckes, Magnitude, diversity, capacities and dimensions of metric spaces, Potential Anal. 42 no. 2 (2015) 549-572.

[18] E. M. Stein, Singular integrals and differentiability properties of functions, Princeton Mathematical Series, No. 30. Princeton University Press, Princeton, N.J. 1970

[19] S. Willerton, Heuristic and computer calculations for the magnitude of metric spaces, arXiv:0910.5500 1 [math.MG]

[20] S. Willerton, On the magnitude of spheres, surfaces and other homogeneous spaces, Geom. Dedicata 168 (2014) 291-310.

[21] The online encyclopaedia of integer sequences, https://oeis.org/

Juan Antonio Barceló, ETSi de Caminos, Canales y Puertos, Universidad Politécnica DE MAdRID, 28040 MAdRID, Spain

E-mail address: juanantonio.barcelo@upm.es

Anthony Carbery, School of Mathematics and Maxwell Institute for Mathematical Sciences, University of Edinburgh, JCMB, Peter Guthrie Tait Road, King's Buildings, Mayfield Road, Edinburgh, EH9 3FD, Scotland.

E-mail address: A.Carbery@ed.ac.uk 